\title{
Bisimulation Minimisations for Boolean Equation Systems
}

\author{
Jeroen Keiren and Tim A.C. Willemse \\ Department of Mathematics and Computer Science, \\ Technische Universiteit Eindhoven, \\ P.O. Box 513, 5600 MB Eindhoven, The Netherlands
}

\begin{abstract}
Boolean equation systems (BESs) have been used to encode several complex verification problems, including model checking and equivalence checking. We introduce the concepts of strong bisimulation and idempotence-identifying bisimulation for BESs, and we prove that these can be used for minimising BESs prior to solving these. Our results show that large reductions of the BESs may be obtained efficiently. Minimisation is rewarding for BESs with non-trivial alternations: the time required for solving the original BES mostly exceeds the time required for quotienting plus the time for solving the quotient. Furthermore, we provide a verification example that demonstrates that bisimulation minimisation of a process prior to encoding the verification problem on that process as a BES can be arbitrarily less effective than minimising the BES that encodes the verification problem.
\end{abstract}

\section{Introduction}

Model checking suffers from the state space explosion problem. Minimising the state space prior to model checking is a well-known strategy for combating this explosion problem, but it is not always obvious that it actually pays to do so in practice. Based on complexity arguments, one can expect that bisimulation minimisation speeds-up model checking for the modal $\mu$-calculus, as in general, the latter requires time exponential in the alternation depth with the size of the state space as root of the exponent (note that some fragments of the $\mu$-calculus can be decided in polynomial time).

The weakest minimisation that is uniformly permitted in the setting of $\mu$-calculus model checking is a minimisation with respect to strong bisimulation, as the logic can distinguish states up-to bisimilarity. On a case-by-case basis, one can, of course, employ weaker process equivalence relations such as trace equivalence, but judging whether this is the case can require a deep understanding of the system, the formula and process theory. In any case, among all process equivalence relations, bisimulation has the most appealing theoretical time complexity $(\mathcal{O}(m \log n)$ with $m$ the size of the transition relation and $n$ the number of states); in practice, it is rivalled only by some weaker bisimulation relations such as branching bisimulation, with time complexity $\mathcal{O}(m n)$.

The downside of using strong bisimulation for minimising a state space prior to verification is that the minimising capabilities of strong bisimulations are often disappointing. One can improve on this by applying abstractions to the state space prior to applying a bisimulation minimisation, but this suffers from the problems that it (1) requires human intellect, and (2) requires different abstractions each time new properties are verified.

We tackle problems 1 and 2 by employing an intermediate framework, viz., Boolean equation systems (BESs). This framework allows one to encode a variety of verification problems, including the modal $\mu$-calculus model checking problem (see, e.g., [10]). Note that the encoded verification problem can subsequently be answered by computing the solution to the resulting equation system. Solving Boolean equation systems again requires time exponential in the alternation depth of the equation system, with the size of the equation system as root of this exponent (the size of the equation system is polynomial in the size of the state space); efficient algorithms for solving equation systems are based on algorithms for solving Parity Games, a framework closely related to BESs. 
Instead of performing minimisation of the state space before encoding the verification problem as a Boolean equation system, we apply minimisation techniques on the equation system itself. For these minimisation techniques, we take inspiration from the notion of bisimulation for state spaces. More concretely, we define two notions of bisimulation for Boolean equation systems, viz., strong bisimulation and idempotence-identifying bisimulation; the latter is tailored specifically to Boolean equation systems and, as far as we are aware, appears to have no natural counterpart in other settings. We show that both notions are equivalence relations and can be used for quotienting; both are computable in time $\mathcal{O}(m \log n)$, with $m$ the size of all right-hand sides of the equations and $n$ the number of equations. Moreover, we prove that strong bisimulation is strictly finer than idempotence-identifying bisimulation, which again is strictly finer than solution equivalence (the latter basically is an equivalence relation based on the local solution of Boolean equation systems, which is typically sufficient for verification). We illustrate that state space minimisation prior to encoding a model checking problem into a Boolean equation system can be arbitrarily less effective than minimising the Boolean equation system that is the result of the encoding.

The advantage of minimisation within the framework of Boolean equation systems is that it does not require human intellect for applying abstractions in order to work. This is because abstraction is taken care of by the encoding to Boolean equation systems. As both bisimulation minimisations respect the solution equivalence, applying minimisations to the Boolean equation system cannot adversely affect the validity of the verification effort, so the approach is fail-safe.

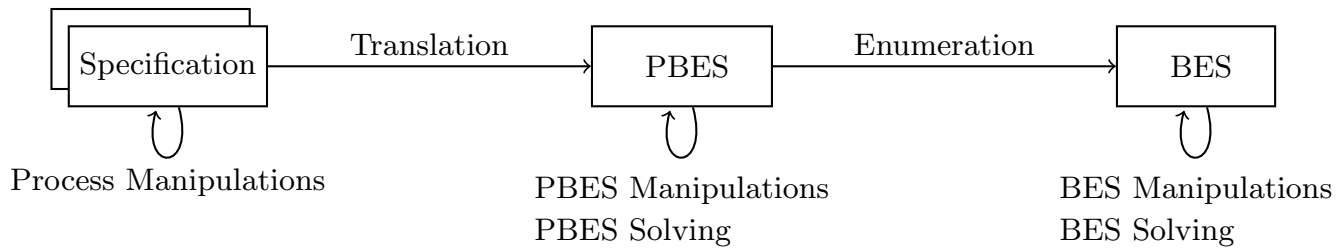

Fig. 1. Verification approach using parameterised Boolean equation systems.

Our bisimulation minimisation techniques provide essential contributions to the framework of parameterised Boolean equation systems (PBESs) [6]. The latter are basically high-level, symbolic descriptions of Boolean equation systems, which are obtained automatically through encodings of (a variety of) verification problems using symbolic system descriptions as input, see Fig. 1. Various solution strategies for PBESs have been described, among which one finds reductions of PBESs to BESs (see e.g. [11], indicated by the arrow linking PBES and BES in Fig. 1). Efficiently minimising the size of the resulting BESs, prior to solving them, is desirable. Observe that the PBES approach to verification avoids generating state spaces altogether; consequently, state space minimisation is not an option in the first place.

We demonstrate the practical value of our approach using a series of experiments that are set in the PBES framework. We rely on state-of-the-art algorithms for solving BESs. The results of these experiments show that indeed, the minimisation of the Boolean equation systems prior to solving is highly rewarding in general: the time required for minimisation is mostly significantly smaller than the time required for solving the unreduced system. The experiments show that in most cases, strong bisimulation and idempotence-identifying bisimulation do not yield significant differences in minimisation capabilities, but, given the more pleasing characteristics of idempotence-identifying bisimulation, the latter is favoured.

Related work. The use of minimisation techniques in combination with verification has been studied in various settings, with mixed results. For LTL verification, Fisler and Vardi [3] show that the total time spent on minimising and verifying exceeds the verification time of the original state space. This can be explained in part, because of the on-the-fly nature of LTL model checking, which does not always require a full construction of a state space. In contrast, in a probabilistic setting, 
Katoen et al. [8] demonstrate that, like in our setting, minimisation mostly pays. Minimisation techniques for Boolean equation systems have received little, if any, attention. In the setting of Parity Games, one finds at least the notion of strong bisimulation and several weaker simulation variants, see [5], but no comparable notion such as idempotence-identifying bisimulation. To the best of our knowledge, in the latter setting, no practical experiments have been conducted using such equivalence relations.

Structure. Section 2 introduces the framework of equation systems, and in Section 3, we define the notions of strong bisimulation and idempotence-identifying bisimulation. In Section 4, we describe experiments conducted using our minimisation methods. The contributions of this paper, and future work are summarised in Section 5.

\section{Preliminaries}

Boolean equation systems are basically finite sequences of least and greatest fixed point equations, where each right-hand side of an equation is a proposition in positive form. For an excellent treatment of the associated theory, we refer to [10]; in the remainder of this section, we focus on the theory required for understanding the results obtained in this paper.

\subsection{Boolean Equation Systems: Syntax and Semantics}

Definition 1. A Boolean equation system $(\mathrm{BES}) \mathcal{E}$ is defined by the following grammar:

$$
\begin{aligned}
& \mathcal{E} \quad::=\epsilon \mid(\sigma X=f) \mathcal{E} \\
& f, g::=c|X| f \vee g \mid f \wedge g
\end{aligned}
$$

where $\epsilon$ is the empty BES, $\sigma \in\{\mu, \nu\}$ is a fixed point symbol, $X$ is a proposition variable taken from some set $\mathcal{X}, f$ and $g$ are proposition formulae and $c \in\{$ true, false $\}$.

For any equation system $\mathcal{E}$, the set of bound proposition variables, bnd $(\mathcal{E})$, is the set of variables occurring at the left-hand side of some equation in $\mathcal{E}$. The set of occurring proposition variables, $\operatorname{occ}(\mathcal{E})$, is the set of variables occurring at the right-hand side of some equation in $\mathcal{E}$; for a specific equation we write rhs $(X)$ to indicate the set of proposition variables occurring in $X$ 's equation.

$$
\begin{array}{ll}
\operatorname{bnd}(\epsilon) \triangleq \emptyset & \operatorname{bnd}((\sigma X=f) \mathcal{E}) \triangleq \operatorname{bnd}(\mathcal{E}) \cup\{X\} \\
\operatorname{occ}(\epsilon) \triangleq \emptyset & \operatorname{occ}((\sigma X=f) \mathcal{E}) \triangleq \operatorname{occ}(\mathcal{E}) \cup \operatorname{occ}(f)
\end{array}
$$

where $\operatorname{occ}(f)$ is defined inductively as follows:

$$
\begin{array}{cc}
\operatorname{occ}(c) \triangleq \emptyset & \operatorname{occ}(X) \triangleq\{X\} \\
\operatorname{occ}(f \vee g) \triangleq \operatorname{occ}(f) \cup \operatorname{occ}(g) & \operatorname{occ}(f \wedge g) \triangleq \operatorname{occ}(f) \cup \operatorname{occ}(g)
\end{array}
$$

For an equation $\sigma X=f$, we set $\operatorname{rhs}(X) \triangleq \operatorname{occ}(f)$. As usual, for reasons of consistency, we consider only equation systems $\mathcal{E}$ in which every proposition variable occurs at the left-hand side of at most one equation of $\mathcal{E}$. We define an ordering $\leqslant$ on bound variables of an equation system $\mathcal{E}$, where $X \triangleq X^{\prime}$ indicates that the equation for $X$ precedes the equation for $X^{\prime}$. We say an $\mathcal{E}$ is closed whenever $\operatorname{occ}(\mathcal{E}) \subseteq \operatorname{bnd}(\mathcal{E})$.

Proposition formulae are interpreted in a context of an environment $\eta: \mathcal{X} \rightarrow \mathbb{B}$. For an arbitrary environment $\eta$, we write $\eta[X:=b]$ for the environment $\eta$ in which the proposition variable $X$ has Boolean value $b$ (note that, for brevity, we do not formally distinguish between a semantic Boolean value and its representation by true and false; likewise, for the operands $\wedge$ and $\vee$ ). 
Definition 2. Let $\eta: \mathcal{X} \rightarrow \mathbb{B}$ be an environment. The interpretation $\llbracket f \rrbracket \eta$ maps a proposition formula $f$ to true or false:

$$
\begin{array}{cc}
\llbracket c \rrbracket \eta \triangleq c & \llbracket X \rrbracket \eta \triangleq \eta(X) \\
\llbracket f \vee g \rrbracket \eta \triangleq \llbracket f \rrbracket \eta \vee \llbracket g \rrbracket \eta & \llbracket f \wedge g \rrbracket \eta \triangleq \llbracket f \rrbracket \eta \wedge \llbracket g \rrbracket \eta
\end{array}
$$

The solution of a BES, given an environment $\eta$, is inductively defined as follows:

$$
\begin{aligned}
& \llbracket \epsilon \rrbracket \eta \quad \triangleq \eta \\
& \llbracket(\sigma X=f) \mathcal{E} \rrbracket \eta \triangleq \begin{cases}\llbracket \mathcal{E} \rrbracket(\eta[X:=\llbracket f \rrbracket(\llbracket \mathcal{E} \rrbracket \eta[X:=\text { false }])]) & \text { if } \sigma=\mu \\
\llbracket \mathcal{E} \rrbracket(\eta[X:=\llbracket f \rrbracket(\llbracket \mathcal{E} \rrbracket \eta[X:=\text { true }])]) & \text { if } \sigma=\nu\end{cases}
\end{aligned}
$$

We refer to computing $\llbracket \rrbracket \eta$ as solving $\mathcal{E}$. Closed equation systems enjoy the property that the solution to the equation system is independent of the environment in which it is defined, i.e., for all environments $\eta, \eta^{\prime}$, we have $\llbracket \mathcal{E} \rrbracket \eta(X)=\llbracket \mathcal{E} \rrbracket \eta^{\prime}(X)$ for all $X \in \operatorname{bnd}(\mathcal{E})$. For this reason, we henceforth omit the environment in our considerations and we write $\llbracket \mathcal{E} \rrbracket$, and $\llbracket \mathcal{E} \rrbracket(X)$ instead.

The disjunction and conjunctions in a proposition formula satisfy the standard rules of logic. For instance, both are semantically idempotent, commutative and associative.

Definition 3. Let $\mathcal{E}$ be an equation system. We say that $\mathcal{E}$ is in standard recursive form (SRF) if the right-hand sides $f$ of every one of its equations can be written using the following grammar:

$$
f::=X|\bigvee F| \bigwedge F, \quad \text { where } F \subseteq \mathcal{X} \text {, with }|F|>0
$$

where the interpretation is given by the following rules:

$$
\llbracket X \rrbracket \eta \triangleq \eta(X) \quad \llbracket \bigvee F \rrbracket \triangleq \bigvee\{\eta(X) \mid X \in F\} \quad \llbracket \bigwedge F \rrbracket \triangleq \bigwedge\{\eta(X) \mid X \in F\}
$$

We introduce the following terminology.

- The function $\mathrm{op}(X)$ for a given equation $(\sigma X=f)$ in $S R F$, returns whether $f$ is conjunctive $(\wedge)$, disjunctive $(\vee)$ or neither $(\perp)$.

- An equation system in SRF is disjunctive if it does not contain any conjunctions

- An equation system in SRF is conjunctive if it does not contain any disjunctions.

Let $\mathcal{B}$ denote the set of all closed equation systems in SRF, and $\mathcal{B}_{\wedge}$ and $\mathcal{B}_{\vee}$ denote the conjunctive and disjunctive subsets, respectively. Observe that $\mathcal{B} \neq \mathcal{B}_{\vee} \cup \mathcal{B}_{\wedge}$. In this paper, we are only concerned with equation systems in $\mathcal{B}$.

Remark 1. Every closed equation system $\mathcal{E}$ can be rewritten to an equation system $\tilde{\mathcal{E}} \in \mathcal{B}$ such that $\llbracket \mathcal{E} \rrbracket \eta(X)=\llbracket \tilde{\mathcal{E}} \rrbracket \eta(X)$ for all $X \in \operatorname{bnd}(\mathcal{E})$, i.e., the transformation to SRF preserves the solution of bound variables. This transformation leads to a polynomial blow-up of the original equation system. In [13], the theory outlined in this paper is generalised to arbitrary closed equation systems. An important observation there is that restricting to equation systems in SRF is only beneficial to the minimisations studied in this paper.

We write $\mathcal{E} \leqslant \mathcal{E}^{\prime}$ iff $\mathcal{E}$ can be derived from $\mathcal{E}^{\prime}$ by strengthening each right-hand side of $\mathcal{E}^{\prime}$. Let $\sqsubseteq$ be an ordering on environments, defined by $\eta \sqsubseteq \eta^{\prime}$ iff for all $X, \eta(X)$ implies $\eta^{\prime}(X) ; \mathcal{E} \leqslant \mathcal{E}^{\prime}$ implies $\llbracket \mathcal{E} \rrbracket \sqsubseteq \llbracket \mathcal{E}^{\prime} \rrbracket$. Mader proves a powerful property for equation systems in $\mathcal{B}$ (see Proposition 3.36 of $[10])$, which we paraphrase below:

Lemma 1. Let $\mathcal{E} \in \mathcal{B}$. There are equation systems $\mathcal{E}_{\wedge} \in \mathcal{B}_{\wedge}$ and $\mathcal{E}_{\vee} \in \mathcal{B}_{\vee}$, such that $\mathcal{E}_{\wedge} \leqslant \mathcal{E}$ and $\mathcal{E} \leqslant \mathcal{E}_{\vee}$ and $\llbracket \mathcal{E} \rrbracket=\llbracket \mathcal{E}_{\wedge} \rrbracket=\llbracket \mathcal{E}_{\vee} \rrbracket$. 


\subsection{Dependency Graphs}

The alternation hierarchy of an equation system, and the derived notion of the rank of an equation, can be thought of as the number of syntactic alternations of fixed point signs occurring in the equation system. Note that the alternation hierarchy is not the same as the alternation depth: the latter is a measure for the complexity of an equation system, measuring the degree of mutual alternating dependencies, and can be smaller than the alternation hierarchy; it is, however, harder to define and compute.

Definition 4. Let $\mathcal{E}$ be an arbitrary equation system. The rank of some $X \in \operatorname{bnd}(\mathcal{E})$, denoted $\operatorname{rank}(X)$, is defined as $\operatorname{rank}(X)=\operatorname{rank}_{\nu, X}(\mathcal{E})$, where $\operatorname{rank}_{\nu, X}(\mathcal{E})$ is defined inductively as follows:

$$
\begin{aligned}
\operatorname{rank}_{\sigma, X}(\epsilon) & =0 \\
\operatorname{rank}_{\sigma, X}\left(\left(\sigma^{\prime} Y=f\right) \mathcal{E}\right) & = \begin{cases}0 & \text { if } \sigma=\sigma^{\prime} \text { and } X=Y \\
\operatorname{rank}_{\sigma, X}(\mathcal{E}) & \text { if } \sigma=\sigma^{\prime} \text { and } X \neq Y \\
1+\operatorname{rank}_{\sigma^{\prime}, X}\left(\left(\sigma^{\prime} Y=f\right) \mathcal{E}\right) & \text { if } \sigma \neq \sigma^{\prime}\end{cases}
\end{aligned}
$$

The alternation hierarchy ah $(\mathcal{E})$ is the difference between the maximum and the minimum of the ranks of the equations of $\mathcal{E}$. Observe that $\operatorname{rank}(X)$ is odd iff $X$ is defined in a least fixed-point equation.

A derived notion of an equation system $\mathcal{E} \in \mathcal{B}$ is its dependency graph $\mathcal{G}_{\mathcal{E}}$, which is defined as a structure $\langle V, \rightarrow, r, l\rangle$, where:

$-V=\operatorname{bnd}(\mathcal{E})$ is the set of states;

$-\rightarrow \subseteq V \times V$ is the transition relation, defined as $X \rightarrow Y$ iff $Y \in \operatorname{rhs}(X)$;

$-r: V \rightarrow \mathbb{N}$ is the rank function, defined as $r(X)=\operatorname{rank}(X)$

$-l: V \rightarrow\{\wedge, \vee, \perp\}$ is the logic function, defined as $l(X)=\mathrm{op}(X)$.

An alternative characterisation of the solution of a particular proposition variable $X$ in an equation system $\mathcal{E} \in \mathcal{B}_{\vee} \cup \mathcal{B}_{\wedge}$ is obtained through the use of the dependency graph $\mathcal{G}_{\mathcal{E}}$. We first define the notion of a $\nu$-dominated lasso.

Definition 5. Let $\mathcal{E} \in \mathcal{B}$ and let $\mathcal{G}_{\mathcal{E}}$ be its dependency graph. A lasso through $\mathcal{G}_{\mathcal{E}}$, starting in a node $X$, is a finite path $\left\langle X_{0}, X_{1}, \ldots, X_{n}\right\rangle$, satisfying $X_{0}=X, X_{n}=X_{j}$ for some $j \leqslant n$, and for each $1 \leqslant i \leqslant n, X_{i-1} \rightarrow X_{i}$. A lasso is said to be $\nu$-dominated if $\min \left\{r\left(X_{i}\right) \mid j \leqslant i \leqslant n\right\}$ is even; otherwise, it is $\mu$-dominated.

The following lemma is loosely based on lemmata taken from Keinänen (see lemmata 40 and 41 in $[9])$.

Lemma 2. Let $\mathcal{E} \in \mathcal{B}$ and let $\mathcal{G}_{\mathcal{E}}$ be its dependency graph. Let $X \in \operatorname{bnd}(\mathcal{E})$. Then:

1. $\mathcal{E} \in \mathcal{B}_{\vee}$ implies $\llbracket \mathcal{E} \rrbracket(X)=$ true iff some lasso starting in $X$ in $\mathcal{G}_{\mathcal{E}}$ is $\nu$-dominated;

2. $\mathcal{E} \in \mathcal{B}_{\wedge}$ implies $\llbracket \mathcal{E} \rrbracket(X)=$ true iff all lassoes starting in $X$ in $\mathcal{G}_{\mathcal{E}}$ are $\nu$-dominated;

Proof. We only consider the first statement; the proof of the second statement is analogous. Observe that when the proposition variable on the cycle of the lasso has an even lowest rank, it is a greatest fixed-point equation $\nu X^{\prime}=f$, with $X^{\prime} \leqslant Y$ for all other equations $\sigma Y=g$ that are on the cycle. This follows from the fact that these have higher ranks. Gauß elimination [10] allows one to substitute $g$ for $Y$ in the equation for $X^{\prime}$, yielding $\nu X^{\prime}=f[Y:=g]$. Since, ultimately, $X^{\prime}$ depends on $X^{\prime}$ again, this effectively enables one to rewrite $\nu X^{\prime}=f$ to $\nu X^{\prime}=f^{\prime} \vee X^{\prime}$. The solution to $\nu X^{\prime}=f^{\prime} \vee X^{\prime}$ is easily seen to be $X^{\prime}=$ true. Since all equations on the lasso are disjunctive, this solution ultimately propagates through the entire lasso, leading to $X=$ true.

Conversely, observe that due to Lemma 1 , there is an equation system $\mathcal{E}^{\prime}$ consisting entirely of equations of the form $\sigma X^{\prime}=X^{\prime \prime}$, with the additional property that $\llbracket \mathcal{E} \rrbracket=\llbracket \mathcal{E}^{\prime} \rrbracket$. In $\mathcal{E}^{\prime}$, the answer to $X$ can only be true if it depends at some point on some $\nu X^{\prime}=X^{\prime \prime}$, where ultimately, $X^{\prime \prime}$ again depends on $X^{\prime}$, leading to a cycle in the dependency graph with even lowest rank. 


\section{Equivalences}

The alternation hierarchy, the number of equations and the complexity of the right-hand sides of these equations account for the computational complexity of the solution for an equation system. Efficient techniques for reducing one or more of these is of the utmost importance. An important step in this direction is to consider equivalence relations for equation systems. An obvious equivalence relation on equation systems is based on the concept of solution for an equation system.

Definition 6. Let $\mathcal{E}, \mathcal{E}^{\prime} \in \mathcal{B}$. We say equations for $X$ and $Y$ are solution equivalent, denoted $X \equiv Y$, if $\llbracket \mathcal{E} \rrbracket(X)=\llbracket \mathcal{E}^{\prime} \rrbracket(Y)$; we say $\mathcal{E}$ and $\mathcal{E}^{\prime}$ are solution equivalent, denoted $\mathcal{E} \equiv \mathcal{E}^{\prime}$, if their first equations are solution equivalent.

In a possible lattice of equivalence relations on equation systems, $\equiv$ is the coarsest equivalence relation of interest. Deciding $\equiv$ is in NP $\cap$ co-NP. Let $\mathcal{E}$ be an equation system. We abbreviate ah $(\mathcal{E})$ by $d$, the number of equations in $\mathcal{E}$ by $n$, the cumulative size of the right-hand sides in $\mathcal{E}$ by $m$ and the size of $\mathcal{E}$ is $m+n$. Algorithms for computing the solution (and thereby deciding $\equiv)$ are, e.g., Small Progress Measures [7] which runs in $\mathcal{O}\left(d m\left(\frac{n}{d}\right)^{\lceil d / 2\rceil}\right)$, bigstep [15] which runs in $\mathcal{O}\left(m n^{\frac{1}{3} d}\right)$, and Gauß Elimination [10] which runs in $\mathcal{O}\left(2^{m+n}\right)$.

In the remainder of this section, we define and study two finer equivalences, viz., strong bisimilarity and idempotence-identifying bisimilarity, the latter being a subtle adaptation of bisimilarity for equation systems which has, to the best of our knowledge, no natural counterpart in other domains.

\subsection{Strong Bisimilarity}

Strong bisimilarity (hereafter referred to as bisimilarity) for equation systems is inspired by the corresponding notion in domains such as process theory and modal logic. While bisimilarity has never been defined for equation systems, it is somehow known in the related framework of Parity Games, see [2].

Definition 7. Let $\mathcal{E}, \mathcal{E}^{\prime} \in \mathcal{B}$. A relation $R \subseteq \operatorname{bnd}(\mathcal{E}) \times \operatorname{bnd}\left(\mathcal{E}^{\prime}\right)$ is said to be a bisimulation if, whenever $X R Y$, then:

$-\operatorname{rank}(X)=\operatorname{rank}(Y)$;

$-\mathrm{op}(X)=\mathrm{op}(Y)$

- for all $U \in \operatorname{occ}(X)$, there is a $V \in \operatorname{occ}(Y)$, such that $U R V$;

- for all $V \in \operatorname{occ}(Y)$, there is a $U \in \operatorname{occ}(X)$, such that $U R V$;

We say equations for $X$ and $Y$ are bisimilar, denoted $X \sim Y$, if there exists a bisimulation relation $R$ such that $X R Y$; we say $\mathcal{E}$ and $\mathcal{E}^{\prime}$ are bisimilar, denoted $\mathcal{E} \sim \mathcal{E}^{\prime}$, if their first equations are bisimilar.

Proposition 1. Bisimilarity is an equivalence relation over $\mathcal{B}$.

Proof. Reflexivity and symmetry follow immediately. For transitivity, we observe that if $X \sim Y$ (due to some $R$ with $X R Y$ ) and $Y \sim Z$ (due to some $S$ with $Y S Z$ ), then $X \sim Z$ is due to $X S \circ R Y$, which is a bisimulation relation.

Let $\operatorname{rhs}(X)_{/ R}=\left\{\left[X^{\prime}\right]_{/ R} \mid X^{\prime} \in \operatorname{rhs}(X)\right\}$ denote the set of classes $\left[X^{\prime}\right]_{/ R}$ in the right hand side of $X$ with respect to a relation $R$. Note that bisimilarity $\sim$ is the union of all bisimulation relations, and, as such, is again a bisimulation relation. Bisimilarity can be used to minimise an equation system via quotienting. This is achieved by constructing an equation for each equivalence class, using both the rank and the logical operand of the equivalence class as building blocks. Observe that each pair of bisimilar equations $\sigma X=f$ and $\sigma^{\prime} X^{\prime}=f^{\prime}$ satisfies $\operatorname{rank}(X)=\operatorname{rank}\left(X^{\prime}\right)$ and $\mathrm{op}(f)=\mathrm{op}\left(f^{\prime}\right)$.

Definition 8. Let $\mathcal{E} \in \mathcal{B}$. The quotient of $\mathcal{E}$, denoted $\mathcal{E}_{/ \sim}$ is an equation system consisting of equations $\sigma_{i} C_{i}=f_{i}$, for $i \in[1 . . n]$, where: 
- $C_{i} \in \operatorname{bnd}(\mathcal{E})_{/ \sim}$, i.e., each $C_{i} \subseteq \operatorname{bnd}(\mathcal{E})$ is exactly one equivalence class of $\mathcal{E}$;

- Let $X \in C_{i}$ and set $F \triangleq \operatorname{rhs}(X)_{/ \sim}$. In case op $(X)=\bigwedge$, set $f_{i} \triangleq \Lambda F$. Likewise if op $(X)=\bigvee$, set $f_{i} \triangleq \bigvee \bigvee F$. Set $f_{i} \triangleq C_{j}$ if $F=\left\{C_{j}\right\}$ and op $(X)=\perp$;

- Order equations such that $C_{i} \Vdash C_{j}$ iff there is some $X \in C_{i}$ such that for all $X^{\prime} \in C_{j}$, $\operatorname{rank}(X) \leqslant \operatorname{rank}\left(X^{\prime}\right)$ and $X \Vdash X^{\prime}$ in $\mathcal{E}$.

The above construction satisfies that $\mathcal{E} \sim \mathcal{E}_{/ \sim}$ for arbitrary $\mathcal{E} \in \mathcal{B}$. The following lemma states that quotienting preserves solution equivalence. This guarantees that bisimulation minimisation can be used for solving equation systems.

Lemma 3. Let $\mathcal{E}, \mathcal{E}^{\prime} \in \mathcal{B}$. Then $\mathcal{E}^{\prime} \sim \mathcal{E}_{/ \sim \text { implies }} \mathcal{E}^{\prime} \equiv \mathcal{E}_{/ \sim}$.

Proof. We actually prove a stronger property: each pair of bisimilar equations has the same solution. In particular, this implies that the first equations of $\mathcal{E}^{\prime}$ and $\mathcal{E}_{/ \sim}$ have the same solution. Assume $\mathcal{E}^{\prime} \sim \mathcal{E}_{/ \sim}$. Suppose $\llbracket \mathcal{E}_{/ \sim \rrbracket} \rrbracket(C)=$ true for some $C$. Let $X \in$ bnd $\left(\mathcal{E}^{\prime}\right)$ be such that $X \sim C$; we show that then also $\llbracket \mathcal{E}^{\prime} \rrbracket(X)=$ true.

By Lemma 1 , we know that there is an equation system $\mathcal{F} \in \mathcal{B}_{\wedge}$ such that $\llbracket \mathcal{F} \rrbracket=\llbracket \mathcal{E}_{/ \sim} \rrbracket$. We construct an equation system $\mathcal{E}_{\wedge}^{\prime}$ from $\mathcal{E}^{\prime}$ by replacing each disjunctive equation $\sigma X^{\prime}=\bigvee F$ with $\sigma X^{\prime}=X^{\prime \prime}$, iff there is some equation $\sigma C^{\prime}=C^{\prime \prime}$ in $\mathcal{F}$ originating from $\sigma C^{\prime}=\bigvee F^{\prime}$ in $\mathcal{E}_{/ \sim}$ satisfying (in $\mathcal{E}_{/ \sim}$ ) $X^{\prime} \sim C^{\prime}$ and $X^{\prime \prime} \sim C^{\prime \prime}$. Observe that $\mathcal{E}_{\wedge}^{\prime} \in \mathcal{B}_{\wedge}$ and $\mathcal{E}_{\wedge}^{\prime} \sim \mathcal{F}$.

Next, we show that all paths in $\mathcal{E}_{\wedge}^{\prime}$ and $\mathcal{F}$ coincide. Assume $R$ is a bisimulation relation witnessing $\mathcal{E}_{\wedge}^{\prime} \sim \mathcal{F}$, and $X \sim C$ in particular. Every path in $C$ has a matching path in $X$. Let $\pi=\left\langle C_{0}, C_{1}, \ldots\right\rangle$ be a path starting in $C_{0}=C$. We construct a path $\pi^{\prime}=\left\langle X_{0}, X_{1}, \ldots\right\rangle$ starting in $X_{0}=X$ per induction. Clearly, we have $X_{0} R C_{0}$. Assume $X_{i} R C_{i}$ for some $i$. Because $C_{i+1} \in \operatorname{rhs}\left(C_{i}\right)$, there must be some $X^{\prime} \in \operatorname{rhs}\left(X_{i}\right)$ such that $X^{\prime} R C_{i+1}$. Choose such an $X^{\prime}$, and set $X_{i+1}:=X^{\prime}$. Similarly, every path starting in $X$ can be matched by some path starting in $C$.

By Lemma 2, the fact that $\mathcal{F} \in \mathcal{B}_{\wedge}$ and $\llbracket \mathcal{F} \rrbracket(C)=$ true, we find that all lassoes starting in $C$ are $\nu$-dominated. Hence, also all lassoes in $\mathcal{E}_{\wedge}^{\prime}$, starting in $X$ are $\nu$-dominated. Since $\mathcal{E}_{\wedge}^{\prime} \leqslant \mathcal{E}$ implies $\llbracket \mathcal{E}_{\wedge}^{\prime} \rrbracket \sqsubseteq \llbracket \mathcal{E}^{\prime} \rrbracket$, also $\llbracket \mathcal{E}^{\prime} \rrbracket(X)=$ true.

The case where $\llbracket \mathcal{E}_{/ \sim} \rrbracket(C)=$ false follows the same line of reasoning, constructing an equation system $\mathcal{E}_{\vee}^{\prime}$ from $\mathcal{E}^{\prime}$ based on some $\mathcal{F} \in \mathcal{B}_{\vee}$ with $\llbracket \mathcal{F} \rrbracket=\llbracket \mathcal{E}_{/ \sim} \rrbracket$.

Theorem 1. The relation $\sim$ is strictly finer than $\equiv$.

Proof. The fact that $\sim$ is finer than $\equiv$ follows from $\mathcal{F} \sim \mathcal{F} / \sim$ for all $\mathcal{F}$, the fact that $\sim$ and $\equiv$ are equivalence relations and Lemma 3. Strictness follows from the fact that $(\nu X=Y)(\mu Y=X) \equiv$ $\left(\nu X^{\prime}=Y^{\prime}\right)\left(\nu Y^{\prime}=X^{\prime}\right)$, but not $(\nu X=Y)(\mu Y=X) \sim\left(\nu X^{\prime}=Y^{\prime}\right)\left(\nu Y^{\prime}=X^{\prime}\right)$.

Example 1. Consider the Labelled Transition System given below:

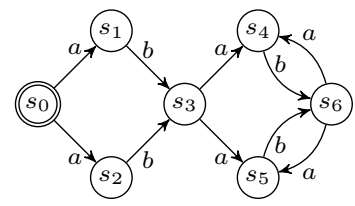

The equation system encoding $s_{0} \models \nu X .[a] \mu Y .\langle b\rangle(Y \vee X)$ is as follows (see, e.g., [10] for an explanation of the syntax and semantics of the $\mu$-calculus, and the encoding of the model checking problem into BES), with its dependency graph depicted below.

$$
\begin{aligned}
& \left(\nu X_{s_{0}}=Y_{s_{1}} \wedge Y_{s_{2}}\right)\left(\nu X_{s_{3}}=Y_{s_{4}} \wedge Y_{s_{5}}\right)\left(\nu X_{s_{6}}=Y_{s_{4}} \wedge Y_{s_{5}}\right) \\
& \left(\mu Y_{s_{1}}=Y_{s_{3}} \vee X_{s_{3}}\right)\left(\mu Y_{s_{2}}=Y_{s_{3}} \vee X_{s_{3}}\right)\left(\mu Y_{s_{3}}=Y_{s_{3}}\right) \\
& \left(\mu Y_{s_{4}}=Y_{s_{6}} \vee X_{s_{6}}\right)\left(\mu Y_{s_{5}}=Y_{s_{6}} \vee X_{s_{6}}\right)\left(\mu Y_{s_{6}}=Y_{s_{6}}\right)
\end{aligned}
$$




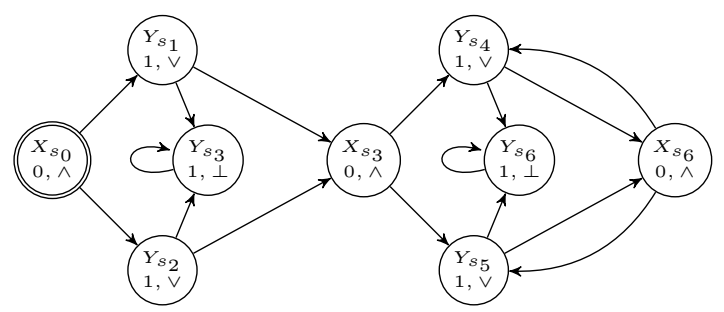

Minimising the equation system module strong bisimulation leads to the following equation system, with the associated dependency graph depicted below.

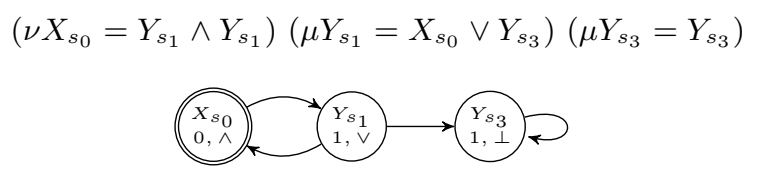

Compared to the original equation system, the minimised equation system is roughly $65 \%$ smaller. Observe that in both the original equation system and the reduced equation system, the solution to $X_{s_{0}}$ is the same, which follows from the fact that both are bisimilar.

\subsection{Idempotence-identifying Bisimulation}

The definition of a quotient for bisimilarity is rather awkward, as equations of the form $\sigma X=$ $X^{\prime} \wedge X^{\prime \prime}$, or $\sigma X=X^{\prime} \vee X^{\prime \prime}$, with $X^{\prime} \sim X^{\prime \prime}$ cannot be minimised further to $\sigma X=X^{\prime}$ (see also the example in the previous section); doing so nevertheless would change the operand of the equation, leading to a violation of $\mathcal{E} \sim \mathcal{E} / \sim$.

From a logical viewpoint, it does not make sense to discriminate between these equations. We observe that a logical operand of an equation is only of importance when it is applied to proposition variables from distinguishable classes; in any other case, the bisimulation relation should be oblivious to the logical operands. These considerations lead us to consider a weaker definition of bisimilarity, called idempotence-identifying bisimilarity, which appears to be more natural in the setting of equation systems.

Definition 9. Let $\mathcal{E}, \mathcal{E}^{\prime} \in \mathcal{B}$. A relation $R \subseteq \operatorname{bnd}(\mathcal{E}) \times \operatorname{bnd}\left(\mathcal{E}^{\prime}\right)$ is said to be an idempotenceidentifying bisimulation if, whenever $X R Y$, then:

$-\operatorname{rank}(X)=\operatorname{rank}(Y)$;

- if op $(X) \neq \mathrm{op}(Y)$ then for all $U \in \operatorname{occ}(X)$ and $V \in \operatorname{occ}(Y): U R V$;

- for each $U \in \operatorname{occ}(X)$ there is a $V \in \operatorname{occ}(Y)$ such that $U R V$;

- for each $V \in \operatorname{occ}(Y)$ there is a $U \in \operatorname{occ}(X)$ such that $U R V$.

We say equations for $X$ and $Y$ are idempotence-identifying bisimilar, denoted $X \sim_{i i} Y$, if there is an idempotence-identifying bisimulation relation $R$ such that $X R Y$; we say $\mathcal{E}$ and $\mathcal{E}^{\prime}$ are idempotence-identifying bisimilar, denoted $\mathcal{E} \sim_{i i} \mathcal{E}^{\prime}$, if their first equations are idempotenceidentifying bisimilar.

Property 1. Let $R, S$ be two idempotence-identifying bisimulation relations over some set of proposition variables $\mathcal{X}$. The union $R \cup S$ is again an idempotence-identifying bisimulation relation.

This property ensures the existence of a maximal idempotence-identifying bisimulation relation, which is the union of all possible idempotence-identifying bisimulation relations.

We next show that idempotence-identifying bisimilarity enjoys the property that it is an equivalence relation (Proposition 2) which is in between bisimilarity and solution equivalence (Theorem 2), and has a natural quotienting operation.

Proposition 2. Idempotence-identifying bisimilarity is an equivalence relation over $\mathcal{B}$. 
Proof. Reflexivity and symmetry follow immediately. We therefore focus on transitivity. Assume that $X \sim_{i i} Y$ and $Y \sim_{i i} Z$ for some $X, Y$ and $Z$. This means that there are idempotence-identifying bisimulation relations $R$ and $S$ such that $X R Y$ and $Y S Z$. Assume that $R$ and $S$ are such. Without loss of generality, assume that these relations are maximal. Then, (i) $X S \circ R Z$, which follows by definition of $S \circ R$ (viz., applying relation $S$ after $R$ ), and, (ii) $S \circ R$ is a idempotenceidentifying bisimulation relation.

Assume that $U S \circ R W$ for some $U, W$. Note that this means there has to be some $V$, such that $U R V$ and $V S W$. We show that $S \circ R$ satisfies the idempotence-identifying bisimulation conditions.

- ad $\operatorname{rank}(U)=\operatorname{rank}(W)$. Observe that we have $U R V$ and $V S W$. From this, both $\operatorname{rank}(U)=$ $\operatorname{rank}(V)$ and $\operatorname{rank}(V)=\operatorname{rank}(W)$ follow, $\operatorname{proving} \operatorname{rank}(U)=\operatorname{rank}(W)$;

- ad op $(U) \neq \operatorname{op}(W)$ implies for all $U^{\prime} \in \operatorname{occ}(U)$ and $W^{\prime} \in \operatorname{occ}(W)$, we have $U^{\prime} S \circ R W^{\prime}$. Assume that op $(U) \neq \mathrm{op}(W)$. Towards a contradiction, assume that the right-hand side of $U$ consists of more than a single class, i.e., $\left|\operatorname{rhs}(U)_{/ R}\right|>1$. Due to maximality of $R$, also $\left|\operatorname{rhs}(V)_{/ R}\right|>1$. Due to maximality of $S,\left|\operatorname{rhs}(V)_{/ S}\right|>1$. Likewise, we derive that $\left|\operatorname{rhs}(W)_{/ S}\right|>$ 1. From $\left|\operatorname{rhs}(V)_{/ R}\right|>1$ and the fact that $R$ is an idempotence-identifying bisimulation relation, the second requirement of idempotence-identifying bisimulation necessarily requires op $(U)=$ $\mathrm{op}(V)$; likewise $\mathrm{op}(V)=\mathrm{op}(W)$. But this contradicts our assumption that $\mathrm{op}(U) \neq \mathrm{op}(W)$. Hence, necessarily $\left|\operatorname{rhs}(U)_{/ R}\right|=1$, i.e., all variables occurring at the right-hand side of the equation for $U$ are related via $R$. Then also $\left|\operatorname{rhs}(V)_{/ R}\right|=1$, and $\left|\operatorname{rhs}(W)_{/ S}\right|=1$. Therefore, all $U^{\prime} \in \operatorname{occ}(U)$ are related to all $W^{\prime} \in \operatorname{occ}(W)$ via $S \circ R$;

- ad for all $U^{\prime} \in \operatorname{occ}(U)$ there is a $W^{\prime} \in \operatorname{occ}(W)$, such that $U^{\prime} S \circ R W^{\prime}$. Let $U^{\prime} \in \operatorname{occ}(U)$. Since $R$ is an idempotence-identifying bisimulation relation, there is some $V^{\prime} \in \operatorname{occ}(V)$ such that $U^{\prime} R V^{\prime}$. Likewise, for this $V^{\prime}$ there is some $W^{\prime} \in \operatorname{occ}(W)$ such that $V^{\prime} S W^{\prime}$. Hence, $U^{\prime} S \circ R W^{\prime}$.

- ad for all $W^{\prime} \in \operatorname{occ}(W)$ there is a $U^{\prime} \in \operatorname{occ}(U)$, such that $U^{\prime} S \circ R W^{\prime}$. Analogous to the previous case.

Quotienting, based on idempotence-identifying bisimulation, requires a subtle modification of the quotienting for bisimulation. In case we are constructing an equation $\sigma_{i} C_{i}=f_{i}$, where $C_{i}$ is again the equivalence class of a set of bisimilar equations, $f_{i}$ is defined as $C_{j}$ in case $\operatorname{rhs}\left(X_{i}\right)_{/ \sim_{i i}}=\left\{C_{j}\right\}$ for all $X_{i} \in C_{i}$. In particular, this avoids introducing awkward equations such as $\sigma_{i} C_{i}=\bigwedge\left\{C_{j}\right\}$. Note that all other cases are in full agreement with Def. 8.

Lemma 4. Let $\mathcal{E}, \mathcal{E}^{\prime} \in \mathcal{B}$. Then $\mathcal{E}^{\prime} \sim_{i i} \mathcal{E}_{/ \sim_{i i}}$ implies $\mathcal{E}^{\prime} \equiv \mathcal{E}_{/ \sim_{i i}}$.

Proof. Let $X \sim_{i i} C$ for some $X$ and $C$. Assume that we have $\llbracket \mathcal{E}_{/ \sim_{i i}} \rrbracket(C)=$ true. We are required to show that $\llbracket \mathcal{E}^{\prime} \rrbracket(X)=$ true. As in the proof of Lemma 3 , one can construct a closed conjunctive equation system $\mathcal{E}_{\wedge}^{\prime}$ based on a conjunctive equation system $\mathcal{F}$ that has the property $\llbracket \mathcal{F} \rrbracket=$ $\llbracket \mathcal{E}_{/ \sim_{i i}} \rrbracket$. Note that a disjunctive equation in $\mathcal{E}^{\prime}$ may be related to a non-disjunctive, non-conjunctive equation $\sigma C=C^{\prime}$ in $\mathcal{E}_{/ \sim_{i i}}$, in which case an arbitrary proposition variable that is idempotenceidentifying bisimilar to $C^{\prime}$ may be chosen. The proof that $\mathcal{E}_{\wedge}^{\prime}$ and $\mathcal{F}$ have the same paths follows the same line of reasoning as in the proof of Lemma 3. The case $\llbracket \mathcal{E}_{/ \sim_{i i}} \rrbracket(C)=$ false is again fully dual.

Theorem 2. We have:

1. the relation $\sim$ is strictly finer than $\sim_{i i}$;

2. the relation $\sim_{i i}$ is strictly finer than $\equiv$;

Proof. We show each property separately.

1. $a d \sim \subseteq \sim_{i i}$; observe that $\sim$ is finer than $\sim_{i i}$ as an immediate consequence of the weakening of the first condition of bisimilarity: for all bisimilar equations defining $X$ and $X^{\prime}$ have the same logical operators, i.e., op $(X)=\mathrm{op}\left(X^{\prime}\right)$. In that case, the second condition for idempotenceidentifying bisimilarity is trivially verified. Strictness follows from the fact that $\nu X=X \sim_{i i}$ $\nu X=X \wedge X$ but not $\nu X=X \sim \nu X=X \wedge X$ 
2. ad $\sim_{i i} \subseteq \equiv$. Follows immediately from $\mathcal{F} \sim_{i i} \mathcal{F} / \sim_{i i}$ for all $\mathcal{F}$, the fact that $\sim_{i i}$ and $\equiv$ are equivalence relations and Lemma 4. For strictness, we refer to the proof of Theorem 1.

The following proposition demonstrates that idempotence-identifying bisimilarity and solution equivalence sometimes coincide.

Proposition 3. Let $\mathcal{E} \in \mathcal{B}$ be of the form $\mathcal{E}_{0} \mathcal{E}_{1} \mathcal{E}_{2}$, with $\mathcal{E}_{1} \in \mathcal{B}$. Suppose $X, X^{\prime} \in \operatorname{bnd}\left(\mathcal{E}_{1}\right)$. Assume $\operatorname{rank}(X)=\operatorname{rank}\left(X^{\prime}\right)$ for all $X, X^{\prime} \in \operatorname{bnd}\left(\mathcal{E}_{1}\right)$. Then $\mathcal{E}_{1 / \sim_{i i}}=\mathcal{E}_{1 / \equiv}$.

Proof. Since $\mathcal{E}_{1} \in \mathcal{B}, \mathcal{E}_{1}$ is closed. Assume $\mathcal{E}_{1}$ has rank $n$ for some $n$. Observe that since $\mathcal{E}_{1}$ is closed, for all $X \in \operatorname{bnd}\left(\mathcal{E}_{1}\right)$, we have $\llbracket \mathcal{E}_{1} \rrbracket(X)=\operatorname{true}$ iff $\operatorname{rank}(X)$ is even. Define $R=\operatorname{bnd}\left(\mathcal{E}_{1}\right) \times \operatorname{bnd}\left(\mathcal{E}_{1}\right)$. It then follows immediately that $R$ is an idempotence-identifying bisimulation relation.

In words, closed sub-equation systems consisting of equations all of the same rank, can be reduced to a single equation. A special case is when $\mathcal{E}_{0}=\mathcal{E}_{2}=\epsilon$, in which case the closed equation system $\mathcal{E}_{1}$ reduces to a single equation. Note that the above result does not hold in the bisimulation setting. In particular, the above result shows that idempotence-identifying bisimilarity can yield a substantially greater reduction, by an arbitrarily large factor, than bisimilarity. The following example illustrates that the same holds when comparing bisimilarity at a process level to idempotence-identifying bisimilarity.

Example 2. Let $N$ be an arbitrary positive natural number. Consider the process described by the following set of recursive processes (using process-algebraic notation):

$$
\left\{S=\sum\{a \cdot X(n) \mid n \leqslant N\}, \quad X(0)=a \cdot X(0)+b \cdot X(0), \quad X(n+1)=b \cdot a \cdot X(n)\right\}
$$

A visualisation of $S$ for $N=3$ is depicted at the right; $S$ consists of $2(N+1)$ states, which cannot be minimised further using strong bisimilarity. Define the following $\mu$-calculus formulae $\phi \triangleq \nu Y .\langle a\rangle([a]$ false $\wedge \nu Z$. $\langle b\rangle\langle a\rangle Z)$. The equation system $\mathcal{E}$, encoding $S \models \phi$ has $N+1$ equations. $\mathcal{E}$ is closed and each of its equations has rank 0 . Following Proposition 3, quotienting of $\mathcal{E}$ yields the equation system $\nu X=X$, for arbitrary $N$. Note that one could reduce the labelled transition system (LTS) underlying $S$ with respect to trace equivalence, yielding an LTS of size 2, which, however, no longer satisfies $\phi$. This is, of course, in general the case, as no process equivalence weaker than strong bisimilarity preserves

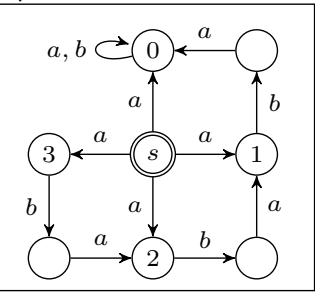
the full modal $\mu$-calculus.

\subsection{Decidability of Bisimilarity and Idempotence-identifying Bisimilarity}

We can use variations of the well known partition refinement algorithm by Paige and Tarjan [12] for deciding both strong bisimilarity and idempotence-identifying bisimilarity, running in $\mathcal{O}(m \log n)$ time. This algorithm iteratively refines the partitioning given a splitting criterion, where a block is split iff there are two equations that are not equivalent. Equivalence of two equations $\sigma X=f$, $\sigma^{\prime} X^{\prime}=f^{\prime}$ given a current partitioning $P$ is decided by the predicate $\mathrm{Eq}_{\sim}\left(\mathrm{P}, \mathrm{X}, \mathrm{X}^{\prime}\right)$ for strong bisimulation and $\mathrm{Eq}_{\sim_{\mathrm{ii}}}\left(\mathrm{P}, \mathrm{X}, \mathrm{X}^{\prime}\right)$ for idempotence-identifying bisimulation, where $\mathrm{Eq}$ is defined as follows.

$$
\begin{aligned}
\mathrm{Eq}_{\sim}\left(\mathrm{P}, \mathrm{X}, \mathrm{X}^{\prime}\right) \triangleq & \operatorname{rank}(X)=\operatorname{rank}\left(X^{\prime}\right) \wedge \operatorname{rhs}(X)_{/ P}=\operatorname{rhs}\left(X^{\prime}\right)_{/ P} \\
& \wedge \operatorname{op}(f)=\operatorname{op}\left(f^{\prime}\right) \\
\mathrm{Eq}_{\sim_{\text {ii }}}\left(\mathrm{P}, \mathrm{X}, \mathrm{X}^{\prime}\right) \triangleq & \operatorname{rank}(X)=\operatorname{rank}\left(X^{\prime}\right) \wedge \operatorname{rhs}(X)_{/ P}=\operatorname{rhs}\left(X^{\prime}\right)_{/ P} \\
& \wedge\left(\left|\operatorname{rhs}(X)_{/ P}\right|=1 \vee \mathrm{op}(f)=\mathrm{op}\left(f^{\prime}\right)\right)
\end{aligned}
$$

That is, in the case of strong bisimulation two equations are equivalent if their ranks and Boolean operators match, and their right-hand sides contain the same classes, whereas for idempotenceidentifying bisimulation the requirement for having the same Boolean operators is lifted in case both equations have only one equivalence class in their right-hand sides. The proofs are similar to the proofs required for decidability of bisimilarity in the setting of transition systems and therefore omitted. 


\section{Experiments}

To test the effectiveness of the minimisations introduced in the previous section, intended mainly to increase efficiency of solving equation systems resulting from typical verification problems, we ran a large set of verification experiments consisting of model checking and process equivalence checking problems. We here present an exhaustive overview of the $>300$ experiments that we have conducted.

Setup. All experiments were run on a workstation consisting of 8 Dual Core ${ }^{1}$ AMD Opteron(tm) Processors running at $2.6 \mathrm{Ghz}$, with $128 \mathrm{~Gb}$ of shared main memory, running a 64 -bit Linux distribution using kernel version 2.6.24. We adapted an off-the-shelf, competitive $\mathrm{C}$ implementation by Blom and Orzan [14] for computing the bisimulation minimisations for LTSs, such that bisimulation and idempotence-identifying bisimulation for BESs can be computed efficiently.

The BESs were solved using a development version of PGSolver tool [4]. ${ }^{2}$ The timings we report have been obtained using the bigstep [15] algorithm, enhanced with a set of heuristics for speeding-up the algorithm, as well as without these enhancements. Note that bigstep outperformed the Small Progress Measures algorithm in all our experiments; both are state-of-the-art algorithms for Parity Games. All BESs were generated from parameterised Boolean equation systems using the mCRL2 tool suite ${ }^{3}$, without generating state spaces first.

\subsection{Process Equivalence Experiments}

We consider the encoding of the branching bisimulation equivalence problem [1], which yields PBESs with alternation depth 2, and, therefore, also BESs of alternation depth 2 . As input to the equivalence checking problem, we used four descriptions of well-studied communications protocols, viz., the one-place buffer (OPB), two variations of the Alternating Bit Protocol (ABP) and the Concurrent Alternating Bit Protocol (CABP). For each protocol, we varied the size of the set of messages $M$ that could be exchanged from $|M|=1,2,4,8,16,32$. Table 1 shows (1) the size of the original BESs in SRF, and (2) the size after reduction using $\sim$ and $\sim_{i i}$; note that the reductions are capable of eliminating the dependency on $|M|$. Table 1 also shows (3) the time for solving the original BES in SRF, and (4) the time required for minimising the BES modulo strong bisimilarity plus solving the resulting BES. Solving times are given both with and without optimisations enabled in PGSolver.

\subsection{Model Checking Experiments}

A second batch of experiments is conducted using the encoding of the $\mu$-calculus model checking problem, yielding $(\mathrm{P}) \mathrm{BESs}$ with alternation depth dependent on the alternation depth of the modal formulae. We use three relatively simple communications protocols, viz., the One Place Buffer (OPB), two variants of the Alternating Bit Protocol (ABP) and the Concurrent Alternating Bit Protocol (CABP). Furthermore we use two complex communications protocols, viz., the Onebit Protocol (OP) and a Sliding Window Protocol with window sizes 1, 2 and $3\left(\mathrm{SWP}_{1}, \mathrm{SWP}_{2}\right.$, $\mathrm{SWP}_{3}$ ). We check for the validity of five modal formulae of increasing complexity. Statistics about the reductions modulo strong bisimulation and idempotence identifying bisimulation as well as solving times for $\mathrm{ABP}_{1}$ are summarised in Table 2; for $\mathrm{ABP}_{2}$ in Table 3; Table 4 shows the results for CABP; Table 5 shows solving times for OPB; solving times for SWP are summarised in Tables 7-9; for OP, they can be found in Table 6. For all protocols, we verified absence of (I) deadlock and (II) livelock, and the possibility to infinitely often (III) receive a certain message, $(\mathrm{IV})$ receive all messages and $(\mathrm{V})$ receive some message if it is infinitely often enabled. $\mathrm{t} / \mathrm{o}$ denotes that the time required for a run exceeded 5 minutes.

\footnotetext{
${ }^{1}$ Note that none of our experiments employ dual-core features.

${ }^{2}$ Obtained from Oliver Friedmann through private communication.

3 See http://www.mcrl2.org, revision 6175 (release branch)
} 


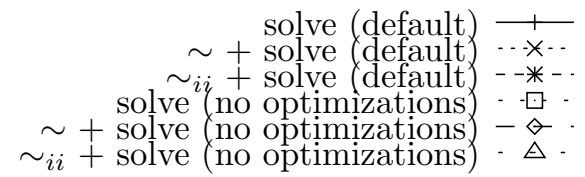

(a) Legend

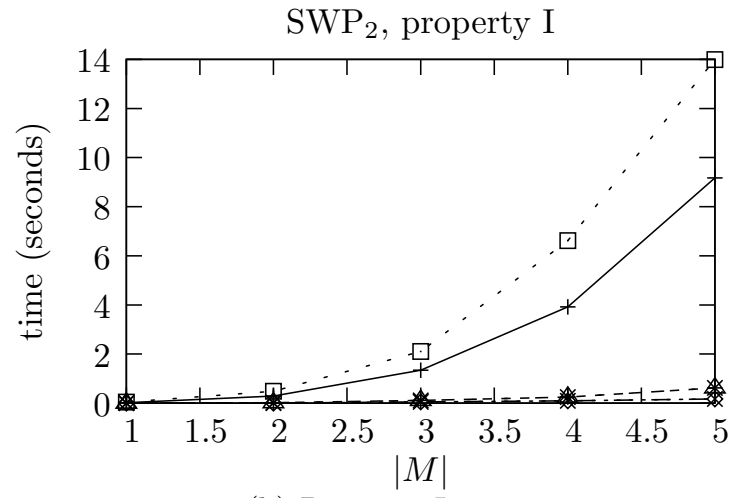

(b) Property I

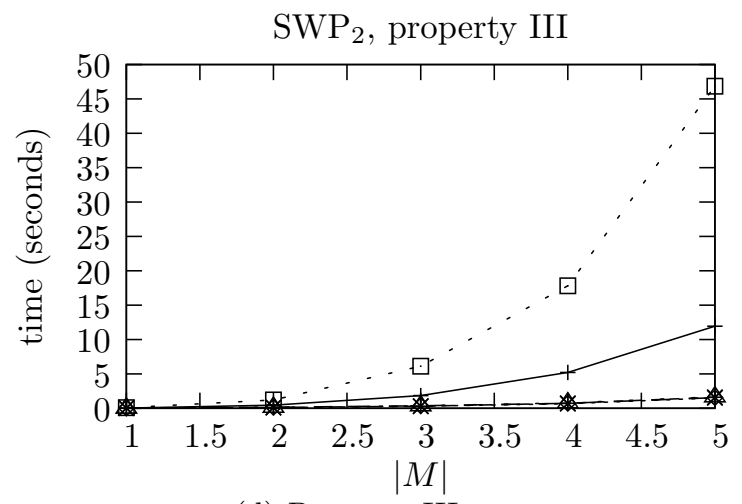

(d) Property III

(c) Property II

$\mathrm{SWP}_{2}$, property IV

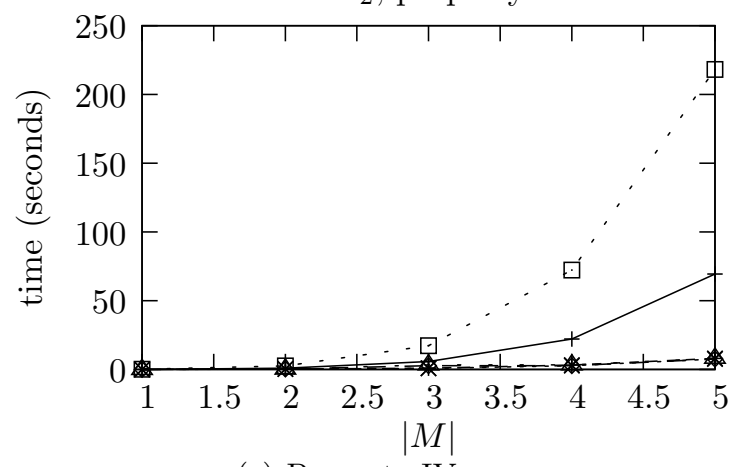

(e) Property IV

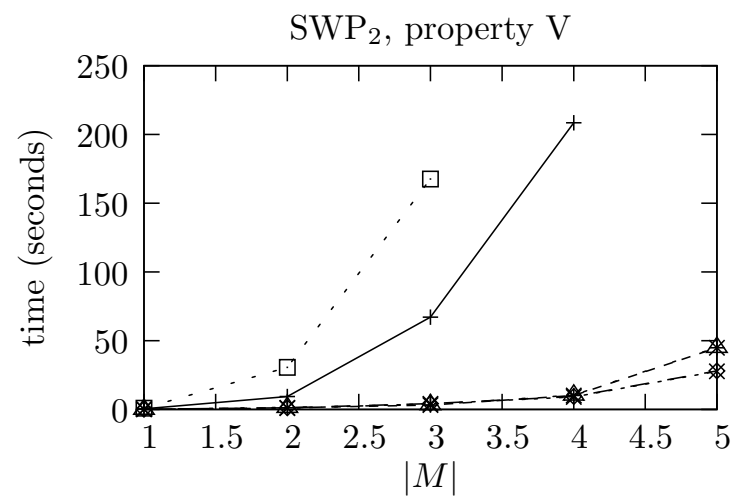

(f) Property V

Fig. 2. Timing results for the sliding window protocol with buffer size 2 (see also Table 8)

\subsection{Discussion}

As can be observed from our experiments, both strong bisimulation and idempotence-identifying bisimulation minimisation show a significant reduction in the size of the BESs. In all cases minimising the BES modulo either of the equivalences and solving the reduced BES outperforms solving the original BES. In addition, reducing modulo strong bisimulation is slightly faster than reducing modulo idempotence-identifying bisimulation in general. This is expected to be a consequence of the additional checks that need to be carried out in an implementation of idempotence-identifying bisimulation, combined with the small difference in size between the systems reduced modulo strong bisimulation and idempotence-identifying bisimulation. 
As an enlightning demonstration of the results, consider the graphs for $\mathrm{SWP}_{2}$ presented in Figure 2. These graphs clearly demonstrate the dramatic effect using bisimulation minimisation prior to solving a BES. As a sidenote also the effect of having optimisation in the solver enabled versus not having optimisations enabled is displayed nicely.

Based on the results of our experiments we believe that in practice a bisimulation reduction should be performed prior to solving the BES.

\section{Conclusions}

In this paper, we have defined two equivalence relations for BESs, viz., strong bisimilarity and idempotence-identifying bisimilarity. The former takes inspiration from the definition of bisimilarity in settings such as process theory and logic. The latter is a modification of strong bisimilarity which is more natural in the setting of equation systems, and has more pleasing properties when used in quotienting.

Our experiments using our two bisimulation minimisation algorithms indeed confirm that enormous reductions are quite commonplace. Moreover, our time measurements show that it pays to minimise before solving: the time required for minimising is more than made up for by the time gained in solving the minimised equation system.

Several topics remain to be investigated. Among these is the investigation of weaker equivalence relations for equation systems. Here, stuttering equivalence (or, equivalently, branching bisimulation) may serve as a source of inspiration, both because of its attractive computational complexity, and because of its capability of achieving far greater minimisations than strong bisimilarity. A related topic that we are currently pursuing is the development of proof theory for parameterised Boolean equation systems [6] based on bisimilarity. We are convinced this will lead to more concise proofs in this setting.

\section{References}

1. T. Chen, B. Ploeger, J. van de Pol, and T.A.C. Willemse. Equivalence checking for infinite systems using Parameterized Boolean Equation Systems. In Proc. of CONCUR 200\%, volume 4703 of LNCS, pages 120-135. Springer, 2007.

2. K. Etessami, T. Wilke, and R.A. Schuller. Fair simulation relations, parity games, and state space reduction for büchi automata. SIAM J. Comput., 34(5):1159-1175, 2005.

3. K. Fisler and M.Y. Vardi. Bisimulation minimization and symbolic model checking. Formal Methods in System Design, 21(1):39-78, 2002.

4. O. Friedmann and M. Lange. Solving parity games in practice. In Proc. of ATVA, volume 5799 of LNCS, pages 182-196. Springer, 2009.

5. C. Fritz and T. Wilke. Simulation relations for alternating parity automata and parity games. In Developments in Language Theory, volume 4036 of Lecture Notes in Computer Science, pages 59-70. Springer, 2006.

6. J.F. Groote and T.A.C. Willemse. Parameterised Boolean Equation Systems. Theor. Comput. Sci, 343(3):332-369, 2005.

7. M. Jurdziński. Small progress measures for solving parity games. In Proc. of STACS '00, LNCS, pages 290-301. Springer, 2000.

8. J.-P. Katoen, T. Kemna, I.S. Zapreev, and D.N. Jansen. Bisimulation minimisation mostly speeds up probabilistic model checking. In Proc. of TACAS'0\%, volume 4424 of $L N C S$, pages 76-92. Springer, 2007.

9. M.K. Keinänen. Solving Boolean Equation Systems. PhD thesis, Helsinki University of Technology, 2006.

10. A. Mader. Verification of Modal Properties Using Boolean Equation Systems. PhD thesis, Technische Universität München, 1997.

11. R. Mateescu and D.Thivolle. A model checking language for concurrent value-passing systems. In Proc. of FM 2008, volume 5014 of LNCS. Springer, 2008.

12. R. Paige and R.E. Tarjan. Three partition refinement algorithms. SIAM J. Comput., 16(6), 1987. 
13. M.A. Reniers and T.A.C. Willemse. Analysis of Boolean equation systems through structure graphs. EPTCS, 2009. To appear.

14. S.Blom and S.Orzan. Distributed state space minimization. Int. J. STTT, 7(3), 2005.

15. S. Schewe. Solving parity games in big steps. In Proc. of FSTTCS 200\%, volume 4855 of LNCS, pages 449-460. Springer, 2007. 


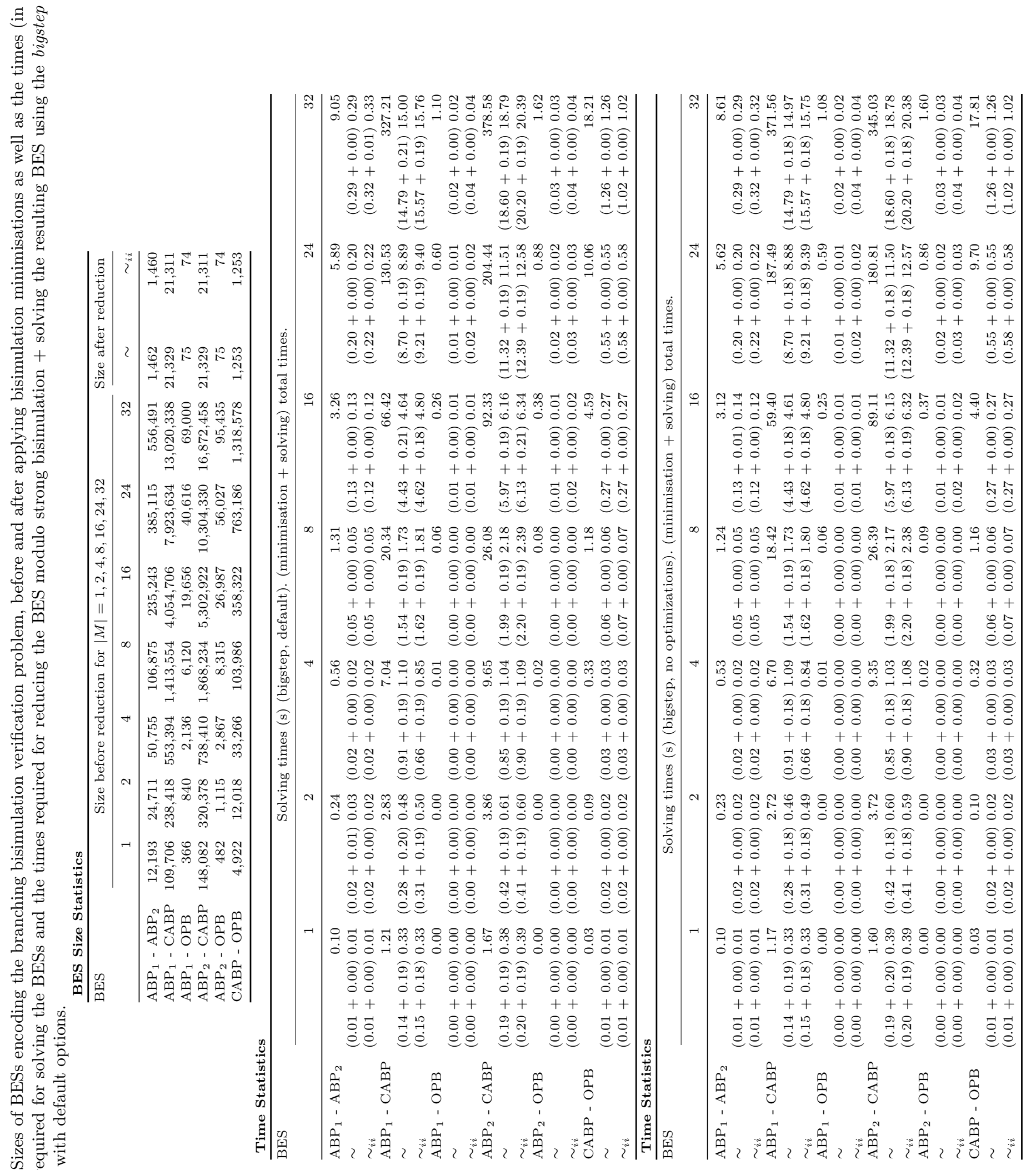


Table 2. $\mathrm{ABP}_{1}$

\begin{tabular}{|c|c|c|c|c|c|c|c|c|c|c|c|c|c|}
\hline \multirow[b]{2}{*}{$|M|$} & \multicolumn{3}{|c|}{ BES size } & \multicolumn{5}{|c|}{ Solving times (bigstep, default) } & \multicolumn{5}{|c|}{ Solving times (bigstep, no optimizations) } \\
\hline & original & $\sim$ & $\sim_{i i}$ & original & & $\sim$ & & $\sim_{i i}$ & original & & $\sim$ & & $\sim_{i i}$ \\
\hline \multicolumn{14}{|c|}{ Property I } \\
\hline 1 & 86 & 16 & 2 & 0.00 & $(0.00+0.00)$ & 0.00 & $(0.00+0.00)$ & 0.00 & 0.00 & $(0.00+0.00)$ & 0.00 & $(0.00+0.00)$ & 0.00 \\
\hline 2 & 168 & 35 & 2 & 0.00 & $(0.00+0.00)$ & 0.00 & $(0.00+0.00)$ & 0.00 & 0.00 & $(0.00+0.00)$ & 0.00 & $(0.00+0.00)$ & 0.00 \\
\hline 3 & 250 & 35 & 2 & 0.00 & $(0.00+0.00)$ & 0.00 & $(0.00+0.00)$ & 0.00 & 0.00 & $(0.00+0.00)$ & 0.00 & $(0.00+0.00)$ & 0.00 \\
\hline 4 & 332 & 35 & 2 & 0.00 & $(0.00+0.00)$ & 0.00 & $(0.00+0.00)$ & 0.00 & 0.00 & $(0.00+0.00)$ & 0.00 & $(0.00+0.00)$ & 0.00 \\
\hline 5 & 414 & 35 & 2 & 0.00 & $(0.00+0.00)$ & 0.00 & $(0.00+0.00)$ & 0.00 & 0.00 & $(0.00+0.00)$ & 0.00 & $(0.00+0.00)$ & 0.00 \\
\hline 6 & 496 & 35 & 2 & 0.00 & $(0.00+0.00)$ & 0.00 & $(0.00+0.00)$ & 0.00 & 0.00 & $(0.00+0.00)$ & 0.00 & $(0.00+0.00)$ & 0.00 \\
\hline 7 & 578 & 35 & 2 & 0.00 & $(0.00+0.00)$ & 0.00 & $(0.00+0.00)$ & 0.00 & 0.00 & $(0.00+0.00)$ & 0.00 & $(0.00+0.00)$ & 0.00 \\
\hline 8 & 660 & 35 & 2 & 0.00 & $(0.00+0.00)$ & 0.00 & $(0.00+0.00)$ & 0.00 & 0.00 & $(0.00+0.00)$ & 0.00 & $(0.00+0.00)$ & 0.00 \\
\hline 16 & 1,316 & 35 & 2 & 0.00 & $(0.00+0.00)$ & 0.00 & $(0.00+0.00)$ & 0.00 & 0.00 & $(0.00+0.00)$ & 0.00 & $(0.00+0.00)$ & 0.00 \\
\hline 24 & 1,972 & 35 & 2 & 0.00 & $(0.00+0.00)$ & 0.00 & $(0.00+0.00)$ & 0.00 & 0.01 & $(0.00+0.00)$ & 0.00 & $(0.00+0.00)$ & 0.00 \\
\hline 32 & 2,628 & 35 & 2 & 0.00 & $(0.00+0.00)$ & 0.00 & $(0.00+0.00)$ & 0.00 & 0.01 & $(0.00+0.00)$ & 0.00 & $(0.00+0.00)$ & 0.00 \\
\hline
\end{tabular}

Property II

\begin{tabular}{|c|c|c|c|c|c|c|c|c|c|c|c|c|}
\hline 1 & 210 & 47 & 46 & 0.00 & $(0.00+0.00)$ & 0.00 & $(0.00+0.00)$ & 0.00 & 0.00 & $(0.00+0.00)$ & 0.00 & $(0.00+0.00) 0.00$ \\
\hline 2 & 408 & 47 & 46 & 0.00 & $(0.00+0.00)$ & 0.00 & $(0.00+0.00)$ & 0.00 & 0.00 & $(0.00+0.00)$ & 0.00 & $(0.00+0.00) 0.00$ \\
\hline 3 & 606 & 47 & 46 & 0.00 & $(0.00+0.00)$ & 0.00 & $(0.00+0.00)$ & 0.00 & 0.00 & $(0.00+0.00)$ & 0.00 & $(0.00+0.00) 0.00$ \\
\hline 4 & 804 & 47 & 46 & 0.00 & $(0.00+0.00)$ & 0.00 & $(0.00+0.00)$ & 0.00 & 0.00 & $(0.00+0.00)$ & 0.00 & $(0.00+0.00) 0.00$ \\
\hline 5 & 1,002 & 47 & 46 & 0.00 & $(0.00+0.00)$ & 0.00 & $(0.00+0.00)$ & 0.00 & 0.00 & $(0.00+0.00)$ & 0.00 & $(0.00+0.00) 0.00$ \\
\hline 6 & 1,200 & 47 & 46 & 0.00 & $(0.00+0.00)$ & 0.00 & $(0.01+0.00)$ & 0.01 & 0.00 & $(0.00+0.00)$ & 0.00 & $(0.01+0.00) 0.01$ \\
\hline 7 & 1,398 & 47 & 46 & 0.00 & $(0.00+0.00)$ & 0.00 & $(0.00+0.00)$ & 0.00 & 0.00 & $(0.00+0.00)$ & 0.00 & $(0.00+0.00) 0.00$ \\
\hline 8 & 1,596 & 47 & 46 & 0.00 & $(0.00+0.00)$ & 0.00 & $(0.01+0.00)$ & 0.01 & 0.00 & $(0.00+0.00)$ & 0.00 & $(0.01+0.00) 0.01$ \\
\hline 16 & 3,180 & 47 & 46 & 0.01 & $(0.00+0.00)$ & 0.00 & $(0.00+0.00)$ & 0.00 & 0.01 & $(0.00+0.00)$ & 0.00 & $(0.00+0.00) 0.00$ \\
\hline 24 & 4,764 & 47 & 46 & 0.01 & $(0.01+0.00)$ & 0.01 & $(0.00+0.00)$ & 0.00 & 0.02 & $(0.01+0.00)$ & 0.01 & $(0.00+0.00) 0.00$ \\
\hline 32 & 6,348 & 47 & 46 & 0.02 & $(0.00+0.00)$ & 0.00 & $(0.01+0.00)$ & 0.01 & 0.02 & $(0.00+0.00)$ & 0.00 & $(0.01+0.00) 0.01$ \\
\hline
\end{tabular}

Property III

\begin{tabular}{|c|c|c|c|c|c|c|c|c|c|c|c|c|}
\hline 1 & 92 & 40 & 36 & 0.00 & $(0.00+0.00)$ & 0.00 & $(0.00+0.00)$ & 0.00 & 0.00 & $(0.00+0.00) 0.00$ & $(0.00+0.00)$ & 0.00 \\
\hline 2 & 174 & 59 & 54 & 0.00 & $(0.00+0.00)$ & 0.00 & $(0.01+0.00)$ & 0.01 & 0.00 & $(0.00+0.00) 0.00$ & $(0.01+0.00)$ & 0.01 \\
\hline 3 & 256 & 59 & 54 & 0.00 & $(0.00+0.00)$ & 0.00 & $(0.00+0.00)$ & 0.00 & 0.00 & $(0.00+0.00) 0.00$ & $(0.00+0.00)$ & 0.00 \\
\hline 4 & 338 & 59 & 54 & 0.00 & $(0.00+0.00)$ & 0.00 & $(0.00+0.00)$ & 0.00 & 0.00 & $(0.00+0.00) 0.00$ & $(0.00+0.00)$ & 0.00 \\
\hline 5 & 420 & 59 & 54 & 0.00 & $(0.00+0.00)$ & 0.00 & $(0.00+0.00)$ & 0.00 & 0.00 & $(0.00+0.00) 0.00$ & $(0.00+0.00)$ & 0.00 \\
\hline 6 & 502 & 59 & 54 & 0.00 & $(0.00+0.00)$ & 0.00 & $(0.00+0.00)$ & 0.00 & 0.00 & $(0.00+0.00) 0.00$ & $(0.00+0.00)$ & 0.00 \\
\hline 7 & 584 & 59 & 54 & 0.00 & $(0.00+0.00)$ & 0.00 & $(0.01+0.00)$ & 0.01 & 0.00 & $(0.00+0.00) 0.00$ & $(0.01+0.00)$ & 0.01 \\
\hline 8 & 666 & 59 & 54 & 0.00 & $(0.00+0.00)$ & 0.00 & $(0.00+0.00)$ & 0.00 & 0.00 & $(0.00+0.00) 0.00$ & $(0.00+0.00)$ & 0.00 \\
\hline 16 & 1,322 & 59 & 54 & 0.00 & $(0.00+0.00)$ & 0.00 & $(0.00+0.00)$ & 0.00 & 0.02 & $(0.00+0.00) 0.00$ & $(0.00+0.00)$ & 0.00 \\
\hline 24 & 1,978 & 59 & 54 & 0.00 & $(0.00+0.00)$ & 0.00 & $(0.02+0.00)$ & 0.02 & 0.03 & $(0.00+0.00) 0.00$ & $(0.02+0.00)$ & 0.02 \\
\hline 32 & 2,634 & 59 & 54 & 0.01 & $(0.00+0.00)$ & 0.00 & $(0.01+0.00)$ & 0.01 & 0.04 & $(0.00+0.00) 0.00$ & $(0.01+0.00)$ & 0.01 \\
\hline
\end{tabular}

Property IV

\begin{tabular}{|c|c|c|c|c|c|c|c|c|c|c|c|c|}
\hline 1 & 94 & 42 & 38 & 0.00 & $(0.01+0.00)$ & 0.01 & $(0.00+0.00)$ & 0.00 & 0.00 & $(0.01+0.00)$ & 0.01 & $(0.00+0.00) 0.00$ \\
\hline 2 & 349 & 62 & 56 & 0.00 & $(0.00+0.00)$ & 0.00 & $(0.00+0.00)$ & 0.00 & 0.00 & $(0.00+0.00)$ & 0.00 & $(0.00+0.00) 0.00$ \\
\hline 3 & 768 & 62 & 56 & 0.00 & $(0.00+0.00)$ & 0.00 & $(0.01+0.00)$ & 0.01 & 0.01 & $(0.00+0.00)$ & 0.00 & $(0.01+0.00) 0.01$ \\
\hline 4 & 1,351 & 62 & 56 & 0.00 & $(0.00+0.00)$ & 0.00 & $(0.01+0.00)$ & 0.01 & 0.01 & $(0.00+0.00)$ & 0.00 & $(0.01+0.00) 0.01$ \\
\hline 5 & 2,098 & 62 & 56 & 0.01 & $(0.00+0.00)$ & 0.00 & $(0.01+0.00)$ & 0.01 & 0.03 & $(0.00+0.00)$ & 0.00 & $(0.01+0.00) 0.01$ \\
\hline 6 & 3,009 & 62 & 56 & 0.01 & $(0.00+0.00)$ & 0.00 & $(0.01+0.00)$ & 0.01 & 0.04 & $(0.00+0.00)$ & 0.00 & $(0.01+0.00) 0.01$ \\
\hline 7 & 4,084 & 62 & 56 & 0.01 & $(0.01+0.00)$ & 0.01 & $(0.01+0.00)$ & 0.01 & 0.06 & $(0.01+0.00)$ & 0.01 & $(0.01+0.00) 0.01$ \\
\hline 8 & 5,323 & 62 & 56 & 0.02 & $(0.00+0.00)$ & 0.00 & $(0.01+0.00)$ & 0.01 & 0.09 & $(0.00+0.00)$ & 0.00 & $(0.01+0.00) 0.01$ \\
\hline 16 & 21,139 & 62 & 56 & 0.12 & $(0.01+0.00)$ & 0.01 & $(0.02+0.00)$ & 0.02 & 0.44 & $(0.01+0.00)$ & 0.01 & $(0.02+0.00) 0.02$ \\
\hline 24 & 47,451 & 62 & 56 & 0.30 & $(0.01+0.00)$ & 0.01 & $(0.03+0.00)$ & 0.03 & 1.11 & $(0.01+0.00)$ & 0.01 & $(0.03+0.00) 0.03$ \\
\hline 32 & 84,259 & 62 & 56 & 0.59 & $(0.02+0.00)$ & 0.02 & $(0.05+0.00)$ & 0.05 & 2.10 & $(0.02+0.00)$ & 0.02 & $(0.05+0.00) 0.05$ \\
\hline
\end{tabular}

Property V

\begin{tabular}{|c|c|c|c|c|c|c|c|c|c|c|c|}
\hline 1 & 362 & 145 & 134 & 0.00 & $(0.00+0.00)$ & 0.00 & $(0.00+0.00) 0.00$ & 0.00 & $(0.00+0.00)$ & 0.00 & $(0.00+0.00) 0.00$ \\
\hline 2 & 1,409 & 223 & 210 & 0.00 & $(0.01+0.00)$ & 0.01 & $(0.02+0.00) 0.02$ & 0.00 & $(0.01+0.00)$ & 0.01 & $(0.02+0.00) 0.02$ \\
\hline 3 & 3,144 & 223 & 210 & 0.01 & $(0.01+0.00)$ & 0.01 & $(0.01+0.00) 0.01$ & 0.01 & $(0.01+0.00)$ & 0.01 & $(0.01+0.00) 0.01$ \\
\hline 4 & 5,567 & 223 & 210 & 0.02 & $(0.01+0.00)$ & 0.01 & $(0.01+0.00) 0.01$ & 0.02 & $(0.01+0.00)$ & 0.01 & $(0.01+0.00) 0.01$ \\
\hline 5 & 8,678 & 223 & 210 & 0.04 & $(0.01+0.00)$ & 0.01 & $(0.03+0.00) 0.03$ & 0.05 & $(0.01+0.00)$ & 0.01 & $(0.03+0.00) 0.03$ \\
\hline 6 & 12,477 & 223 & 210 & 0.07 & $(0.02+0.00)$ & 0.02 & $(0.02+0.00) 0.02$ & 0.07 & $(0.02+0.00)$ & 0.02 & $(0.02+0.00) 0.02$ \\
\hline 7 & 16,964 & 223 & 210 & 0.09 & $(0.02+0.00)$ & 0.02 & $(0.02+0.00) 0.02$ & 0.10 & $(0.02+0.00)$ & 0.02 & $(0.02+0.00) 0.02$ \\
\hline 8 & 22,139 & 223 & 210 & 0.13 & $(0.02+0.00)$ & 0.02 & $(0.04+0.00) 0.04$ & 0.14 & $(0.02+0.00)$ & 0.02 & $(0.04+0.00) 0.04$ \\
\hline 16 & 88,307 & 223 & 210 & 0.62 & $(0.03+0.00)$ & 0.03 & $(0.06+0.00) 0.06$ & 0.69 & $(0.03+0.00)$ & 0.03 & $(0.06+0.00) 0.06$ \\
\hline 24 & 198,507 & 223 & 210 & 1.46 & $(0.08+0.00)$ & 0.08 & $(0.13+0.00) 0.13$ & 1.65 & $(0.08+0.00)$ & 0.08 & $(0.13+0.00) 0.13$ \\
\hline 32 & 352,739 & 223 & 210 & 2.78 & $(0.25+0.00)$ & 0.25 & $(0.25+0.00) 0.25$ & 3.06 & $(0.25+0.00)$ & 0.25 & $(0.25+0.00) 0.25$ \\
\hline
\end{tabular}


Table 3. $\mathrm{ABP}_{2}$

\begin{tabular}{|c|c|c|c|c|c|c|c|c|c|c|c|c|c|}
\hline \multirow[b]{2}{*}{$|M|$} & \multicolumn{3}{|c|}{ BES size } & \multicolumn{5}{|c|}{ Solving times (bigstep, default) } & \multicolumn{5}{|c|}{ Solving times (bigstep, no optimizations) } \\
\hline & original & $\sim$ & $\sim_{i i}$ & original & & $\sim$ & & $\sim_{i i}$ & original & & $\sim$ & & $\sim_{i i}$ \\
\hline \multicolumn{14}{|c|}{ Property I } \\
\hline 1 & 113 & 16 & 2 & 0.00 & $(0.00+0.00)$ & 0.00 & $(0.00+0.00)$ & 0.00 & 0.00 & $(0.00+0.00)$ & 0.00 & $(0.00+0.00)$ & 0.00 \\
\hline 2 & 221 & 35 & 2 & 0.00 & $(0.00+0.00)$ & 0.00 & $(0.00+0.00)$ & 0.00 & 0.00 & $(0.00+0.00)$ & 0.00 & $(0.00+0.00)$ & 0.00 \\
\hline 3 & 329 & 35 & 2 & 0.00 & $(0.00+0.00)$ & 0.00 & $(0.00+0.00)$ & 0.00 & 0.00 & $(0.00+0.00)$ & 0.00 & $(0.00+0.00)$ & 0.00 \\
\hline 4 & 437 & 35 & 2 & 0.00 & $(0.00+0.00)$ & 0.00 & $(0.00+0.00)$ & 0.00 & 0.00 & $(0.00+0.00)$ & 0.00 & $(0.00+0.00)$ & 0.00 \\
\hline 5 & 545 & 35 & 2 & 0.00 & $(0.01+0.00)$ & 0.01 & $(0.00+0.00)$ & 0.00 & 0.00 & $(0.01+0.00)$ & 0.01 & $(0.00+0.00)$ & 0.00 \\
\hline 6 & 653 & 35 & 2 & 0.00 & $(0.00+0.00)$ & 0.00 & $(0.00+0.00)$ & 0.00 & 0.00 & $(0.00+0.00)$ & 0.00 & $(0.00+0.00)$ & 0.00 \\
\hline 7 & 761 & 35 & 2 & 0.00 & $(0.00+0.00)$ & 0.00 & $(0.00+0.00)$ & 0.00 & 0.00 & $(0.00+0.00)$ & 0.00 & $(0.00+0.00)$ & 0.00 \\
\hline 8 & 869 & 35 & 2 & 0.00 & $(0.00+0.00)$ & 0.00 & $(0.00+0.00)$ & 0.00 & 0.00 & $(0.00+0.00)$ & 0.00 & $(0.00+0.00)$ & 0.00 \\
\hline 16 & 1,733 & 35 & 2 & 0.00 & $(0.01+0.00)$ & 0.01 & $(0.00+0.00)$ & 0.00 & 0.01 & $(0.01+0.00)$ & 0.01 & $(0.00+0.00)$ & 0.00 \\
\hline 24 & 2,597 & 35 & 2 & 0.00 & $(0.01+0.00)$ & 0.01 & $(0.00+0.00)$ & 0.00 & 0.01 & $(0.01+0.00)$ & 0.01 & $(0.00+0.00)$ & ) 0.00 \\
\hline 32 & 3,461 & 35 & 2 & 0.00 & $(0.00+0.00)$ & 0.00 & $(0.00+0.00)$ & 0.00 & 0.02 & $(0.00+0.00)$ & 0.00 & $(0.00+0.00)$ & 0.00 \\
\hline
\end{tabular}

Property II

\begin{tabular}{|c|c|c|c|c|c|c|c|c|c|}
\hline 1 & 276 & 47 & 46 & 0.00 & $(0.00+0.00) 0.00$ & $(0.00+0.00) 0.00$ & 0.00 & $(0.00+0.00) 0.00$ & $(0.00+0.00) 0.00$ \\
\hline 2 & 536 & 47 & 46 & 0.00 & $0.00) 0.01$ & $(0.00+0.00) 0.00$ & 0.00 & $0.00) 0.01$ & 0.00 \\
\hline 3 & 796 & 47 & 46 & 0.00 & $(0.00+0.00) 0.00$ & $(0.00+0.00) 0.00$ & 0.00 & $(0.00+0.00) 0.00$ & $0.00) 0.00$ \\
\hline 4 & 1,056 & 47 & 46 & 0.00 & $(0.00+0.00) 0.00$ & $(0.00+0.00) 0.00$ & 0.00 & $(0.00+0.00) 0.00$ & $(0.00+0.00) 0.00$ \\
\hline 5 & 1,316 & 47 & 46 & 0.00 & $(0.00+0.00) 0.00$ & $(0.00+0.00) 0.00$ & 0.00 & $(0.00+0.00) 0.00$ & $(0.00+0.00) 0.00$ \\
\hline 6 & 1,576 & 47 & 46 & 0.00 & $(0.00+0.00) 0.00$ & $(0.00+0.00) 0.00$ & 0.00 & $(0.00+0.00) 0.00$ & $(0.00+0.00) 0.00$ \\
\hline 7 & 1,836 & 47 & 46 & 0.00 & $(0.01+0.00) 0.01$ & $(0.01+0.00) 0.01$ & 0.00 & $(0.01+0.00) 0.01$ & $(0.01+0.00) 0.01$ \\
\hline 8 & 2,096 & 47 & 46 & 0.00 & $(0.00+0.00) 0.00$ & $(0.00+0.00) 0.00$ & 0.00 & $(0.00+0.00) 0.00$ & $(0.00+0.00) 0.00$ \\
\hline 16 & 4,176 & 47 & 46 & 0.01 & $(0.00+0.00) 0.00$ & $(0.00+0.00) 0.00$ & 0.01 & $(0.00+0.00) 0.00$ & $(0.00+0.00) 0.00$ \\
\hline 24 & 6,256 & 47 & 46 & 0.01 & $(0.00+0.00) 0.00$ & $(0.00+0.00) 0.00$ & 0.02 & $(0.00+0.00) 0.00$ & $(0.00+0.00) 0.00$ \\
\hline 32 & 8,336 & 47 & 46 & 0.02 & $(0.00+0.00) 0.00$ & $(0.01+0.00) 0.01$ & 0.03 & $(0.00+0.00) 0.00$ & $(0.01+0.00) 0.01$ \\
\hline
\end{tabular}

Property III

\begin{tabular}{|c|c|c|c|c|c|c|c|c|c|}
\hline 1 & 119 & 40 & 36 & 0.00 & $(0.00+0.00) 0.00$ & $(0.00+0.00) 0.00$ & 0.00 & $(0.00+0.00) 0.00$ & $(0.00+0.00) 0.00$ \\
\hline 2 & 227 & 59 & 54 & 0.00 & $(0.00+0.00) 0.00$ & $(0.00+0.00) 0.00$ & 0.00 & $(0.00+0.00) 0.00$ & $(0.00+0.00) 0.00$ \\
\hline 3 & 335 & 59 & 54 & 0.00 & $(0.01+0.00) 0.01$ & $(0.00+0.00) 0.00$ & 0.00 & $(0.01+0.00) 0.01$ & $(0.00+0.00) 0.00$ \\
\hline 4 & 443 & 59 & 54 & 0.00 & $(0.01+0.00) 0.01$ & $(0.00+0.00) 0.00$ & 0.00 & $(0.01+0.00) 0.01$ & $(0.00+0.00) 0.00$ \\
\hline 5 & 551 & 59 & 54 & 0.00 & $(0.00+0.00) 0.00$ & $(0.00+0.00) 0.00$ & 0.00 & $(0.00+0.00) 0.00$ & $(0.00+0.00) 0.00$ \\
\hline 6 & 659 & 59 & 54 & 0.00 & $(0.00+0.00) 0.00$ & $(0.01+0.00) 0.01$ & 0.01 & $(0.00+0.00) 0.00$ & $(0.01+0.00) 0.01$ \\
\hline 7 & 767 & 59 & 54 & 0.00 & $(0.01+0.00) 0.01$ & $(0.01+0.00) 0.01$ & 0.01 & $(0.01+0.00) 0.01$ & $(0.01+0.00) 0.01$ \\
\hline 8 & 875 & 59 & 54 & 0.00 & $(0.01+0.00) 0.01$ & $(0.00+0.00) 0.00$ & 0.01 & $(0.01+0.00) 0.01$ & $(0.00+0.00) 0.00$ \\
\hline 16 & 1,739 & 59 & 54 & 0.00 & $(0.00+0.00) 0.00$ & $(0.01+0.00) 0.01$ & 0.03 & $(0.00+0.00) 0.00$ & $(0.01+0.00) 0.01$ \\
\hline 24 & 2,603 & 59 & 54 & 0.01 & $(0.00+0.00) 0.00$ & $(0.01+0.00) 0.01$ & 0.05 & $(0.00+0.00) 0.00$ & $(0.01+0.00) 0.01$ \\
\hline 32 & 3,467 & 59 & 54 & 0.01 & $(0.00+0.00) 0.00$ & $(0.01+0.00) 0.01$ & 0.06 & $(0.00+0.00) 0.00$ & $(0.01+0.00) 0.01$ \\
\hline
\end{tabular}

Property IV

\begin{tabular}{|c|c|c|c|c|c|c|c|c|c|}
\hline & 121 & 42 & 38 & 0.00 & $0.00+0.00) 0.00$ & $(0.00+0.00) 0.00$ & 0.00 & $(0.00+0.00) 0.00$ & $(0.00+0.00) 0.00$ \\
\hline 2 & 455 & 62 & 56 & 0.00 & b0) 0.01 & D0) 0.00 & 0.00 & $(0.01+0.00) 0.01$ & .00) 0.00 \\
\hline 3 & 1,005 & 62 & 56 & 0.00 & 00) 0.00 & $(0.01+0.00) 0.01$ & 0.01 & 00) 0.00 & $.01+0.00) 0.01$ \\
\hline 4 & 1,771 & 62 & 56 & .00 & ) 0.00 & $(0.01+$ & 0.02 & $0) 0.00$ & 00) 0.01 \\
\hline 5 & 2,753 & 62 & 56 & 0.01 & $00) 0.00$ & $(0.01+$ & 0.04 & .00) 0.00 & .00) 0.01 \\
\hline 6 & 3,951 & 62 & 56 & 0.01 & $0.00) 0.01$ & $0.00) 0.01$ & 0.07 & $0.00) 0.01$ & $-0.00) 0.01$ \\
\hline 7 & & 62 & 56 & 0.02 & 0.00 & $(0$ & 0.09 & 0.00 & 00) 0.01 \\
\hline & 6,995 & 62 & 56 & 02 & 0.00 & 0.02 & 0.12 & 0.00 & 0.02 \\
\hline 6 & 27,811 & 62 & 56 & 0.17 & $(0.02+0.00) 0.02$ & 0.03 & 0.64 & $(0.02+0.00) 0.02$ & $(0.03+0.00) 0.03$ \\
\hline 24 & 62,451 & 62 & 56 & 0.49 & $(0.02+0.00) 0.02$ & $(0.04+0.00) 0.04$ & 1.60 & $(0.02+0.00) 0.02$ & $(0.04+0.00) 0.04$ \\
\hline 2 & 110,915 & 62 & 56 & 0.78 & $(0.03+0.00) 0.03$ & $(0.07+0.00) 0.07$ & 2.95 & $(0.03+0.00) 0.03$ & $(0.07+0.00) 0.07$ \\
\hline
\end{tabular}

Property V

\begin{tabular}{|c|c|c|c|c|c|c|c|c|c|}
\hline 1 & 476 & 145 & 134 & 0.00 & $(0.01+0.00) 0.01$ & $(0.01+0.00) 0.01$ & 0.00 & $(0.01+0.00) 0.01$ & $(0.01+0.00) 0.01$ \\
\hline 2 & 1,851 & 223 & 210 & 0.00 & $(0.00+0.00) 0.00$ & $00) 0.01$ & 0.00 & $(0.00+0.00) 0.00$ & $0.00) 0.01$ \\
\hline 3 & 4,128 & 223 & 210 & 0.01 & $(0.01+0.00) 0.01$ & $(0.01+0.00) 0.01$ & 0.01 & $(0.01+0.00) 0.01$ & $0.00) 0.01$ \\
\hline 4 & 7,307 & 223 & 210 & 0.03 & $(0.01+0.00) 0.01$ & $(0.02+0.00) 0.02$ & 0.03 & $(0.01+0.00) 0.01$ & $(0.02+0.00) 0.02$ \\
\hline 5 & 11,388 & 223 & 210 & 0.05 & $(0.01+0.00) 0.01$ & $(0.02+0.00) 0.02$ & 0.06 & $(0.01+0.00) 0.01$ & $(0.02+0.00) 0.02$ \\
\hline 6 & 16,371 & 223 & 210 & 0.10 & $(0.01+0.00) 0.01$ & $(0.02+0.00) 0.02$ & 0.10 & $(0.01+0.00) 0.01$ & $(0.02+0.00) 0.02$ \\
\hline 7 & 22,256 & 223 & 210 & 0.12 & $(0.01+0.00) 0.01$ & $(0.02+0.00) 0.02$ & 0.15 & $(0.01+0.00) 0.01$ & $(0.02+0.00) 0.02$ \\
\hline 8 & 29,043 & 223 & 210 & 0.17 & $(0.03+0.00) 0.03$ & $(0.03+0.00) 0.03$ & 0.19 & $(0.03+0.00) 0.03$ & $(0.03+0.00) 0.03$ \\
\hline 16 & 115,811 & 223 & 210 & 0.81 & $(0.07+0.00) 0.07$ & $(0.07+0.00) 0.07$ & 0.91 & $(0.07+0.00) 0.07$ & $-0.00) 0.07$ \\
\hline 24 & 260,307 & 223 & 210 & 2.07 & $(0.10+0.00) 0.10$ & $(0.26+0.00) 0.26$ & 2.23 & $(0.10+0.00) 0.10$ & $(0.26+0.00) 0.26$ \\
\hline 32 & 462,531 & 223 & 210 & 3.74 & $(0.20+0.00) 0.20$ & $(0.35+0.00) 0.35$ & 4.25 & $(0.20+0.00) 0.20$ & $(0.35+0.00) 0.35$ \\
\hline
\end{tabular}


Table 4. CABP

\begin{tabular}{|c|c|c|c|c|c|c|c|c|c|c|c|c|c|}
\hline \multirow[b]{2}{*}{$|M|$} & \multicolumn{3}{|c|}{ BES size } & \multicolumn{5}{|c|}{ Solving times (bigstep, default) } & \multicolumn{5}{|c|}{ Solving times (bigstep, no optimizations) } \\
\hline & original & $\sim$ & $\sim_{i i}$ & original & & $\sim$ & & $\sim_{i i}$ & original & & $\sim$ & & $\sim_{i i}$ \\
\hline \multicolumn{14}{|c|}{ Property I } \\
\hline 1 & 986 & 5 & 2 & 0.00 & $(0.00+0.00)$ & 0.00 & $(0.00+0.00)$ & 0.00 & 0.00 & $(0.00+0.00)$ & 0.00 & $(0.00+0.00)$ & 0.00 \\
\hline 2 & 2,098 & 5 & 2 & 0.00 & $(0.00+0.00)$ & 0.00 & $(0.00+0.00)$ & 0.00 & 0.00 & $(0.00+0.00)$ & 0.00 & $(0.00+0.00)$ & 0.00 \\
\hline 3 & 3,386 & 5 & 2 & 0.00 & $(0.00+0.00)$ & 0.00 & $(0.00+0.00)$ & 0.00 & 0.01 & $(0.00+0.00)$ & 0.00 & $(0.00+0.00)$ & 0.00 \\
\hline 4 & 4,850 & 5 & 2 & 0.01 & $(0.00+0.00)$ & 0.00 & $(0.00+0.00)$ & 0.00 & 0.02 & $(0.00+0.00)$ & 0.00 & $(0.00+0.00)$ & 0.00 \\
\hline 5 & 6,490 & 5 & 2 & 0.01 & $(0.00+0.00)$ & 0.00 & $(0.00+0.00)$ & 0.00 & 0.02 & $(0.00+0.00)$ & 0.00 & $(0.00+0.00)$ & 0.00 \\
\hline 6 & 8,306 & 5 & 2 & 0.02 & $(0.00+0.00)$ & 0.00 & $(0.00+0.00)$ & 0.00 & 0.03 & $(0.00+0.00)$ & 0.00 & $(0.00+0.00)$ & 0.00 \\
\hline 7 & 10,298 & 5 & 2 & 0.02 & $(0.00+0.00)$ & 0.00 & $(0.00+0.00)$ & 0.00 & 0.04 & $(0.00+0.00)$ & 0.00 & $(0.00+0.00)$ & 0.00 \\
\hline 8 & 12,466 & 5 & 2 & 0.03 & $(0.00+0.00)$ & 0.00 & $(0.00+0.00)$ & 0.00 & 0.06 & $(0.00+0.00)$ & 0.00 & $(0.00+0.00)$ & 0.00 \\
\hline 16 & 36,146 & 5 & 2 & 0.13 & $(0.00+0.00)$ & 0.00 & $(0.02+0.00)$ & 0.02 & 0.22 & $(0.00+0.00)$ & 0.00 & $(0.02+0.00)$ & 0.02 \\
\hline 24 & 71,090 & 5 & 2 & 0.32 & $(0.00+0.00)$ & 0.00 & $(0.02+0.00)$ & 0.02 & 0.47 & $(0.00+0.00)$ & 0.00 & $(0.02+0.00)$ & 0.02 \\
\hline 32 & 117,298 & 5 & 2 & 0.50 & $(0.01+0.00)$ & 0.01 & $(0.04+0.00)$ & 0.04 & 0.81 & $(0.01+0.00)$ & 0.01 & $(0.04+0.00)$ & 0.04 \\
\hline
\end{tabular}

Property II

\begin{tabular}{|c|c|c|c|c|c|c|c|c|c|c|c|}
\hline 1 & 2,130 & 591 & 7 & 0.00 & $(0.00+0.00)$ & 0.00 & $(0.00+0.00) 0.00$ & 0.00 & $(0.00+0.00)$ & 0.00 & $(0.00+0.00) 0.00$ \\
\hline 2 & 4,498 & 591 & 7 & 0.01 & $(0.00+0.00)$ & 0.00 & $(0.00+0.00) 0.00$ & 0.00 & $(0.00+0.00)$ & 0.00 & $(0.00+0.00) 0.00$ \\
\hline 3 & 7,218 & 591 & 7 & 0.01 & $(0.01+0.00)$ & 0.01 & $(0.00+0.00) 0.00$ & 0.01 & $(0.01+0.00)$ & 0.01 & $(0.00+0.00) 0.00$ \\
\hline 4 & 10,290 & 591 & 7 & 0.02 & $(0.01+0.00)$ & 0.01 & $(0.00+0.00) 0.00$ & 0.02 & $(0.01+0.00)$ & 0.01 & $(0.00+0.00) 0.00$ \\
\hline 5 & 13,714 & 591 & 7 & 0.03 & $(0.01+0.00)$ & 0.01 & $(0.01+0.00) 0.01$ & 0.03 & $(0.01+0.00)$ & 0.01 & $(0.01+0.00) 0.01$ \\
\hline 6 & 17,490 & 591 & 7 & 0.04 & $(0.02+0.00)$ & 0.02 & $(0.01+0.00) 0.01$ & 0.05 & $(0.02+0.00)$ & 0.02 & $(0.01+0.00) 0.01$ \\
\hline 7 & 21,618 & 591 & 7 & 0.06 & $(0.01+0.00)$ & 0.01 & $(0.01+0.00) 0.01$ & 0.05 & $(0.01+0.00)$ & 0.01 & $(0.01+0.00) 0.01$ \\
\hline 8 & 26,098 & 591 & 7 & 0.07 & $(0.02+0.00)$ & 0.02 & $(0.01+0.00) 0.01$ & 0.08 & $(0.02+0.00)$ & 0.02 & $(0.01+0.00) 0.01$ \\
\hline 16 & 74,610 & 591 & 7 & 0.26 & $(0.04+0.00)$ & 0.04 & $(0.03+0.00) 0.03$ & 0.25 & $(0.04+0.00)$ & 0.04 & $(0.03+0.00) 0.03$ \\
\hline 24 & 145,650 & 591 & 7 & 0.54 & $(0.07+0.00)$ & 0.07 & $(0.06+0.00) 0.06$ & 0.54 & $(0.07+0.00)$ & 0.07 & $(0.06+0.00) 0.06$ \\
\hline 32 & 239,218 & 591 & 7 & 0.91 & $(0.11+0.00)$ & 0.11 & $(0.10+0.00) 0.10$ & 0.93 & $(0.11+0.00)$ & 0.11 & $(0.10+0.00) 0.10$ \\
\hline
\end{tabular}

Property III

\begin{tabular}{|c|c|c|c|c|c|c|c|c|c|c|c|c|}
\hline 1 & 1,052 & 283 & 283 & 0.00 & $(0.01+0.00)$ & 0.01 & $(0.01+0.00) 0.01$ & 0.01 & $(0.01+0.00)$ & 0.01 & $(0.01+0.00)$ & 0.01 \\
\hline 2 & 2,164 & 403 & 403 & 0.00 & $(0.02+0.00)$ & 0.02 & $(0.02+0.00) 0.02$ & 0.02 & $(0.02+0.00)$ & 0.02 & $(0.02+0.00)$ & 0.02 \\
\hline 3 & 3,452 & 403 & 403 & 0.01 & $(0.02+0.00)$ & 0.02 & $(0.01+0.00) 0.01$ & 0.05 & $(0.02+0.00)$ & 0.02 & $(0.01+0.00)$ & 0.01 \\
\hline 4 & 4,916 & 403 & 403 & 0.01 & $(0.02+0.00)$ & 0.02 & $(0.01+0.00) 0.01$ & 0.07 & $(0.02+0.00)$ & 0.02 & $(0.01+0.00)$ & 0.01 \\
\hline 5 & 6,556 & 403 & 403 & 0.01 & $(0.01+0.00)$ & 0.01 & $(0.01+0.00) 0.01$ & 0.10 & $(0.01+0.00)$ & 0.01 & $(0.01+0.00)$ & 0.01 \\
\hline 6 & 8,372 & 403 & 403 & 0.02 & $(0.01+0.00)$ & 0.01 & $(0.01+0.00) 0.01$ & 0.13 & $(0.01+0.00)$ & 0.01 & $(0.01+0.00)$ & 0.01 \\
\hline 7 & 10,364 & 403 & 403 & 0.03 & $(0.02+0.00)$ & 0.02 & $(0.02+0.00) 0.02$ & 0.17 & $(0.02+0.00)$ & 0.02 & $(0.02+0.00)$ & 0.02 \\
\hline 8 & 12,532 & 403 & 403 & 0.04 & $(0.02+0.00)$ & 0.02 & $(0.02+0.00) 0.02$ & 0.22 & $(0.02+0.00)$ & 0.02 & $(0.02+0.00)$ & 0.02 \\
\hline 16 & 36,212 & 403 & 403 & 0.15 & $(0.04+0.00)$ & 0.04 & $(0.04+0.00) 0.04$ & 0.80 & $(0.04+0.00)$ & 0.04 & $(0.04+0.00)$ & 0.04 \\
\hline 24 & 71,156 & 403 & 403 & 0.38 & $(0.04+0.00)$ & 0.04 & $(0.04+0.00) 0.04$ & 1.69 & $(0.04+0.00)$ & 0.04 & $(0.04+0.00)$ & 0.04 \\
\hline 32 & 117,364 & 403 & 403 & 0.60 & $(0.07+0.00)$ & 0.07 & $(0.07+0.00) 0.07$ & 2.96 & $(0.07+0.00)$ & 0.07 & $(0.07+0.00)$ & 0.07 \\
\hline
\end{tabular}

Property IV

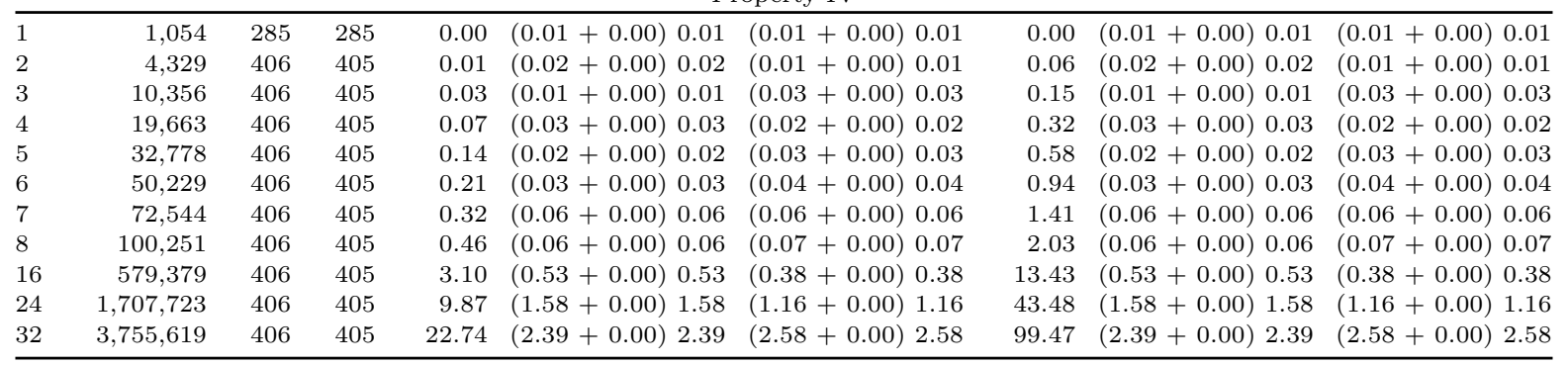

Property V

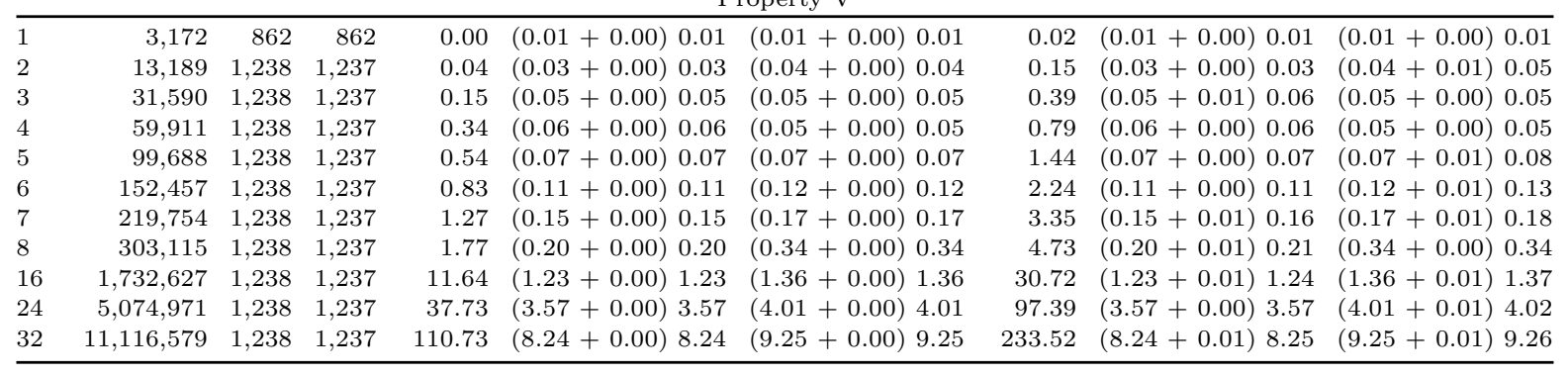


Table 5. OPB

\begin{tabular}{|c|c|c|c|c|c|c|c|c|c|c|c|c|c|}
\hline \multirow[b]{2}{*}{$|M|$} & \multicolumn{3}{|c|}{ BES size } & \multicolumn{5}{|c|}{ Solving times (bigstep, default) } & \multicolumn{5}{|c|}{ Solving times (bigstep, no optimizations) } \\
\hline & original & $\sim$ & $\sim_{i i}$ & original & & $\sim$ & & $\sim_{i i}$ & original & & $\sim$ & & $\sim_{i i}$ \\
\hline \multicolumn{14}{|c|}{ Property I } \\
\hline 1 & 6 & 2 & 2 & 0.00 & $(0.00+0.00)$ & 0.00 & $(0.00+0.00)$ & 0.00 & 0.00 & $(0.00+0.00)$ & 0.00 & $(0.00+0.00)$ & 0.00 \\
\hline 2 & 9 & 5 & 2 & 0.00 & $(0.00+0.00)$ & 0.00 & $(0.00+0.00)$ & 0.00 & 0.00 & $(0.00+0.00)$ & 0.00 & $(0.00+0.00)$ & 0.00 \\
\hline 3 & 12 & 5 & 2 & 0.00 & $(0.00+0.00)$ & 0.00 & $(0.00+0.00)$ & 0.00 & 0.00 & $(0.00+0.00)$ & 0.00 & $(0.00+0.00)$ & 0.00 \\
\hline 4 & 15 & 5 & 2 & 0.00 & $(0.00+0.00)$ & 0.00 & $(0.00+0.00)$ & 0.00 & 0.00 & $(0.00+0.00)$ & 0.00 & $(0.00+0.00)$ & 0.00 \\
\hline 5 & 18 & 5 & 2 & 0.00 & $(0.00+0.00)$ & 0.00 & $(0.00+0.00)$ & 0.00 & 0.00 & $(0.00+0.00)$ & 0.00 & $(0.00+0.00)$ & 0.00 \\
\hline 6 & 21 & 5 & 2 & 0.00 & $(0.00+0.00)$ & 0.00 & $(0.00+0.00)$ & 0.00 & 0.00 & $(0.00+0.00)$ & 0.00 & $(0.00+0.00)$ & 0.00 \\
\hline 7 & 24 & 5 & 2 & 0.00 & $(0.00+0.00)$ & 0.00 & $(0.00+0.00)$ & 0.00 & 0.00 & $(0.00+0.00)$ & 0.00 & $(0.00+0.00)$ & 0.00 \\
\hline 8 & 27 & 5 & 2 & 0.00 & $(0.00+0.00)$ & 0.00 & $(0.00+0.00)$ & 0.00 & 0.00 & $(0.00+0.00)$ & 0.00 & $(0.00+0.00)$ & 0.00 \\
\hline 16 & 51 & 5 & 2 & 0.00 & $(0.00+0.00)$ & 0.00 & $(0.00+0.00)$ & 0.00 & 0.00 & $(0.00+0.00)$ & 0.00 & $(0.00+0.00)$ & 0.00 \\
\hline 24 & 75 & 5 & 2 & 0.00 & $(0.00+0.00)$ & 0.00 & $(0.00+0.00)$ & 0.00 & 0.00 & $(0.00+0.00)$ & 0.00 & $(0.00+0.00)$ & 0.00 \\
\hline 32 & 99 & 5 & 2 & 0.00 & $(0.00+0.00)$ & 0.00 & $(0.00+0.00)$ & 0.00 & 0.00 & $(0.00+0.00)$ & 0.00 & $(0.00+0.00)$ & 0.00 \\
\hline
\end{tabular}

Property II

\begin{tabular}{|c|c|c|c|c|c|c|c|c|c|c|c|c|}
\hline 1 & 14 & 9 & 9 & 0.00 & $(0.00+0.00)$ & 0.00 & $(0.00+0.00)$ & 0.00 & 0.00 & $(0.00+0.00) 0.00$ & $(0.00+0.00)$ & 0.00 \\
\hline 2 & 20 & 9 & 9 & 0.00 & $(0.00+0.00)$ & 0.00 & $(0.00+0.00)$ & 0.00 & 0.00 & $(0.00+0.00) 0.00$ & $(0.00+0.00)$ & 0.00 \\
\hline 3 & 26 & 9 & 9 & 0.00 & $(0.00+0.00)$ & 0.00 & $(0.00+0.00)$ & 0.00 & 0.00 & $(0.00+0.00) 0.00$ & $(0.00+0.00)$ & 0.00 \\
\hline 4 & 32 & 9 & 9 & 0.00 & $(0.00+0.00)$ & 0.00 & $(0.00+0.00)$ & 0.00 & 0.00 & $(0.00+0.00) 0.00$ & $(0.00+0.00)$ & 0.00 \\
\hline 5 & 38 & 9 & 9 & 0.00 & $(0.00+0.00)$ & 0.00 & $(0.00+0.00)$ & 0.00 & 0.00 & $(0.00+0.00) 0.00$ & $(0.00+0.00)$ & 0.00 \\
\hline 6 & 44 & 9 & 9 & 0.00 & $(0.00+0.00)$ & 0.00 & $(0.00+0.00)$ & 0.00 & 0.00 & $(0.00+0.00) 0.00$ & $(0.00+0.00)$ & 0.00 \\
\hline 7 & 50 & 9 & 9 & 0.00 & $(0.00+0.00)$ & 0.00 & $(0.00+0.00)$ & 0.00 & 0.00 & $(0.00+0.00) 0.00$ & $(0.00+0.00)$ & 0.00 \\
\hline 8 & 56 & 9 & 9 & 0.00 & $(0.00+0.00)$ & 0.00 & $(0.00+0.00)$ & 0.00 & 0.00 & $(0.00+0.00) 0.00$ & $(0.00+0.00)$ & 0.00 \\
\hline 16 & 104 & 9 & 9 & 0.00 & $(0.00+0.00)$ & 0.00 & $(0.00+0.00)$ & 0.00 & 0.00 & $(0.00+0.00) 0.00$ & $(0.00+0.00)$ & 0.00 \\
\hline 24 & 152 & 9 & 9 & 0.00 & $(0.00+0.00)$ & 0.00 & $(0.00+0.00)$ & 0.00 & 0.00 & $(0.00+0.00) 0.00$ & $(0.00+0.00)$ & 0.00 \\
\hline 32 & 200 & 9 & 9 & 0.00 & $(0.00+0.00)$ & 0.00 & $(0.00+0.00)$ & 0.00 & 0.00 & $(0.00+0.00) 0.00$ & $(0.00+0.00)$ & 0.00 \\
\hline
\end{tabular}

Property III

\begin{tabular}{|c|c|c|c|c|c|c|c|c|c|c|c|}
\hline 1 & 8 & 8 & 8 & 0.00 & $0.00+0.00)$ & 0.00 & $0.00+0.00) 0.00$ & 0.00 & $(0.00+0.00)$ & 0.00 & $(0.00+0.00) 0.0$ \\
\hline 2 & 11 & 11 & 11 & 0.00 & $.00+0.00)$ & 0.00 & $(0.00+0.00) 0.00$ & 0.00 & $(0.00+0.00)$ & 0.00 & $(0.00+0.00)$ \\
\hline 3 & 14 & 11 & 11 & 0.00 & $(0.00+0.00)$ & 0.00 & $(0.00+0.00) 0.00$ & 0.00 & $(0.00+0.00)$ & 0.00 & $(0.00+0.00) 0.0$ \\
\hline 4 & 17 & 11 & 11 & 0.00 & $(0.00+0.00)$ & 0.00 & $(0.00+0.00) 0.00$ & 0.00 & $0+0$. & 0.00 & $0+0.00) 0$. \\
\hline 6 & 23 & 11 & 11 & 0.00 & $(0.00+0.00)$ & 0.00 & $(0.00+0.00) 0.00$ & 0.00 & $(0.00+0.00)$ & 0.00 & $(0.00+0.00) 0.0$ \\
\hline 7 & 26 & 11 & 11 & 0.00 & $(0.00+0.00)$ & 0.00 & $(0.00+0.00) 0.00$ & 0.00 & $(0.00+0.00)$ & 0.00 & $(0.00+0.00) 0.0$ \\
\hline 8 & 29 & 11 & 11 & 0.00 & $(0.00+0.00)$ & 0.00 & $(0.00+0.00) 0.00$ & 0.00 & $(0.00+0.00)$ & 0.00 & $(0.00+0.00) 0.0$ \\
\hline 32 & 101 & 11 & 11 & 0.00 & $(0.00+0.00)$ & 0.00 & $(0.00+0.00) 0.00$ & 0.00 & $(0.00+0.00)$ & 0.00 & $(0.00+0.00) 0.0$ \\
\hline
\end{tabular}

Property IV

\begin{tabular}{|c|c|c|c|c|c|c|c|c|c|c|c|c|c|}
\hline 1 & 10 & 10 & 10 & 0.00 & $(0.00+0.00)$ & 0.00 & $(0.00+0.00)$ & 0.00 & 0.00 & $(0.00+0.00)$ & 0.00 & $(0.00+0.00)$ & 0.00 \\
\hline 2 & 23 & 14 & 13 & 0.00 & $(0.00+0.00)$ & 0.00 & $(0.00+0.00)$ & 0.00 & 0.00 & $(0.00+0.00)$ & 0.00 & $(0.00+0.00)$ & 0.00 \\
\hline 3 & 42 & 14 & 13 & 0.00 & $(0.00+0.00)$ & 0.00 & $(0.00+0.00)$ & 0.00 & 0.00 & $(0.00+0.00)$ & 0.00 & $(0.00+0.00)$ & 0.00 \\
\hline 4 & 67 & 14 & 13 & 0.00 & $(0.00+0.00)$ & 0.00 & $(0.00+0.00)$ & 0.00 & 0.00 & $(0.00+0.00)$ & 0.00 & $(0.00+0.00)$ & 0.00 \\
\hline 6 & 135 & 14 & 13 & 0.00 & $(0.00+0.00)$ & 0.00 & $(0.00+0.00)$ & 0.00 & 0.00 & $(0.00+0.00)$ & 0.00 & $(0.00+0.00)$ & 0.00 \\
\hline 7 & 178 & 14 & 13 & 0.00 & $(0.00+0.00)$ & 0.00 & $(0.00+0.00)$ & 0.00 & 0.00 & $(0.00+0.00)$ & 0.00 & $(0.00+0.00)$ & 0.00 \\
\hline 8 & 227 & 14 & 13 & 0.00 & $(0.00+0.00)$ & 0.00 & $(0.00+0.00)$ & 0.00 & 0.00 & $(0.00+0.00)$ & 0.00 & $(0.00+0.00)$ & 0.00 \\
\hline 32 & 3,203 & 14 & 13 & 0.00 & $(0.00+0.00)$ & 0.00 & $(0.00+0.00)$ & 0.00 & 0.01 & $(0.00+0.00)$ & 0.00 & $(0.00+0.00)$ & 0.00 \\
\hline
\end{tabular}

Property V

\begin{tabular}{|c|c|c|c|c|c|c|c|c|c|c|c|c|}
\hline 1 & 22 & 22 & 22 & 0.00 & $(0.00+0.00)$ & 0.00 & $(0.00+0.00)$ & 0.00 & 0.00 & $(0.00+0.00) 0.00$ & $(0.00+0.00)$ & 0.00 \\
\hline 2 & 63 & 34 & 33 & 0.00 & $(0.00+0.00)$ & 0.00 & $(0.00+0.00)$ & 0.00 & 0.00 & $(0.00+0.00) 0.00$ & $(0.00+0.00)$ & 0.00 \\
\hline 3 & 126 & 34 & 33 & 0.00 & $(0.00+0.00)$ & 0.00 & $(0.00+0.00)$ & 0.00 & 0.00 & $(0.00+0.00) 0.00$ & $(0.00+0.00)$ & 0.00 \\
\hline 4 & 211 & 34 & 33 & 0.00 & $(0.00+0.00)$ & 0.00 & $(0.00+0.00)$ & 0.00 & 0.00 & $(0.00+0.00) 0.00$ & $(0.00+0.00)$ & 0.00 \\
\hline 5 & 318 & 34 & 33 & 0.00 & $(0.00+0.00)$ & 0.00 & $(0.00+0.00)$ & 0.00 & 0.00 & $(0.00+0.00) 0.00$ & $(0.00+0.00)$ & 0.00 \\
\hline 6 & 447 & 34 & 33 & 0.00 & $(0.00+0.00)$ & 0.00 & $(0.00+0.00)$ & 0.00 & 0.00 & $(0.00+0.00) 0.00$ & $(0.00+0.00)$ & 0.00 \\
\hline 7 & 598 & 34 & 33 & 0.00 & $(0.00+0.00)$ & 0.00 & $(0.00+0.00)$ & 0.00 & 0.00 & $(0.00+0.00) 0.00$ & $(0.00+0.00)$ & 0.00 \\
\hline 8 & 771 & 34 & 33 & 0.00 & $(0.00+0.00)$ & 0.00 & $(0.00+0.00)$ & 0.00 & 0.00 & $(0.00+0.00) 0.00$ & $(0.00+0.00)$ & 0.00 \\
\hline 16 & 2,947 & 34 & 33 & 0.00 & $(0.00+0.00)$ & 0.00 & $(0.00+0.00)$ & 0.00 & 0.01 & $(0.00+0.00) 0.00$ & $(0.00+0.00)$ & 0.00 \\
\hline 24 & 6,531 & 34 & 33 & 0.02 & $(0.00+0.00)$ & 0.00 & $(0.00+0.00)$ & 0.00 & 0.02 & $(0.00+0.00) 0.00$ & $(0.00+0.00)$ & 0.00 \\
\hline 32 & 11,523 & 34 & 33 & 0.04 & $(0.00+0.00)$ & 0.00 & $(0.01+0.00)$ & 0.01 & 0.04 & $(0.00+0.00) 0.00$ & $(0.01+0.00)$ & 0.01 \\
\hline
\end{tabular}


Table 6. OP

\begin{tabular}{|c|c|c|c|c|c|c|c|c|c|c|c|c|}
\hline \multirow[b]{2}{*}{$|M|$} & \multicolumn{3}{|c|}{ BES size } & \multicolumn{4}{|c|}{ Solving times (bigstep, default) } & \multicolumn{5}{|c|}{ Solving times (bigstep, no optimizations) } \\
\hline & original & $\sim$ & $\sim_{i i}$ & original & $\sim$ & & $\sim_{i i}$ & original & & $\sim$ & & $\sim_{i}$ \\
\hline \multicolumn{13}{|c|}{ Property I } \\
\hline 1 & 77,698 & 5 & 2 & 0.29 & $(0.01+0.00) 0.01$ & $(0.03+0.00)$ & 0.03 & 0.76 & $(0.01+0.00$ & 0.01 & $(0.03+0.00)$ & 0.0 \\
\hline 2 & 578,050 & 5 & 2 & 2.68 & $(0.07+0.00) 0.07$ & $(0.21+0.00)$ & 0.21 & 3.94 & $(0.07+0.00$ & 0.07 & $(0.21+0.00)$ & 0.21 \\
\hline 3 & $2,083,394$ & 5 & 2 & 12.41 & $(0.26+0.00) 0.26$ & $(0.75+0.00)$ & 0.75 & 15.95 & $(0.26+0.00$ & 0.26 & $(0.75+0.00)$ & 0.75 \\
\hline 4 & $5,417,986$ & 5 & 2 & 32.40 & $(0.65+0.00) 0.65$ & $(2.05+0.00)$ & 2.05 & 48.70 & $(0.65+0.00$ & 0.65 & $(2.05+0.00)$ & 2.05 \\
\hline \multicolumn{13}{|c|}{ Property II } \\
\hline 1 & 150,210 & 8 & 7 & 0.96 & $(0.04+0.00) 0.04$ & $(0.10+0.00)$ & 0.10 & 0.86 & $(0.04+0.00$ & 0.04 & $(0.10+0.00)$ & 0.10 \\
\hline 2 & $1,100,802$ & 8 & 7 & 5.58 & $(0.45+0.00) 0.45$ & $(0.58+0.00)$ & 0.58 & 4.53 & $(0.45+0.00$ & 0.45 & $(0.58+0.00)$ & 0.58 \\
\hline 3 & $3,933,506$ & 8 & 7 & 20.22 & $(1.65+0.00) 1.65$ & $(2.11+0.00)$ & ) 2.11 & 18.03 & $(1.65+0.00$ & ) 1.65 & $(2.11+0.00)$ & ) 2.11 \\
\hline 4 & $10,172,418$ & 8 & 7 & 73.54 & $(4.79+0.00) 4.79$ & $(5.89+0.00)$ & 5.89 & 54.09 & $(4.79+0.00$ & 4.79 & $(5.89+0.00)$ & 5.88 \\
\hline \multicolumn{13}{|c|}{ Property III } \\
\hline 1 & 89,106 & 13,110 & 13,110 & 0.48 & $(0.17+0.05) 0.22$ & $(0.10+0.04)$ & 0.14 & 0.84 & $(0.17+0.12$ & 0.29 & $(0.10+0.15)$ & 0.25 \\
\hline 2 & 619,010 & 26,171 & 26,171 & 3.84 & $(0.77+0.10) 0.87$ & $(0.82+0.11)$ & 0.93 & 8.68 & $(0.77+0.32$ & 2) 1.09 & $(0.82+0.33)$ & 1.15 \\
\hline 3 & $2,179,826$ & 26,171 & 26,171 & 15.78 & $(2.30+0.11) 2.41$ & $(2.44+0.11)$ & 2.55 & 20.36 & $(2.30+0.33$ & 3) 2.63 & $(2.44+0.33)$ & 2.77 \\
\hline 4 & $5,603,586$ & 26,171 & 26,171 & 46.18 & $(5.60+0.10) 5.70$ & $(6.16+0.11)$ & 6.27 & 59.96 & $(5.60+0.32$ & 5.92 & $(6.16+0.32)$ & 6.48 \\
\hline \multicolumn{13}{|c|}{ Property IV } \\
\hline 1 & 89,108 & 13,112 & 13,112 & 0.85 & $(0.10+0.04) 0.14$ & $(0.11+0.04)$ & ) 0.15 & 1.35 & $(0.10+0.11$ & l) 0.21 & $(0.11+0.11)$ & 0.22 \\
\hline 2 & $1,238,023$ & 26,174 & 26,173 & 9.51 & $(1.50+0.10) 1.60$ & $(1.60+0.10)$ & ) 1.70 & 17.63 & $(1.50+0.33$ & 3) 1.83 & $(1.60+0.33)$ & 1.93 \\
\hline 3 & $6,539,482$ & 26,174 & 26,173 & 47.70 & $(7.00+0.10) 7.10$ & $(7.37+0.10)$ & 7.47 & 64.82 & $(7.00+0.33$ & 3) 7.33 & $(7.37+0.33)$ & 7.70 \\
\hline 4 & $22,414,349$ & 26,174 & 26,173 & 220.33 & $(26.08+0.11) 26.19$ & $(27.24+0.10)$ & 27.34 & 250.54 & $(26.08+0.33)$ & 26.41 & $(27.24+0.32)$ & 27.56 \\
\hline \multicolumn{13}{|c|}{ Property V } \\
\hline 1 & 242,728 & 35,826 & 35,826 & 2.83 & $(0.80+0.17) 0.97$ & $(0.45+0.17)$ & 0.62 & 42.30 & $(0.80+2.20$ & 3.00 & $(0.45+2.20)$ & 2.65 \\
\hline 2 & $3,358,981$ & 71,206 & 71,205 & 31.12 & $(5.24+0.39) 5.63$ & $(5.71+0.38)$ & 6.09 & $\mathrm{t} / \mathrm{o}$ & $(5.24+3.42$ & 8.66 & $(5.71+3.40)$ & 9.11 \\
\hline 3 & $17,664,522$ & 71,206 & 71,205 & 267.34 & $(24.46+0.44) 24.90$ & $(26.08+0.39)$ & 26.47 & $\mathrm{t} / \mathrm{o}$ & $(24.46+3.34)$ & 27.80 & $(26.08+3.37)$ & 29.45 \\
\hline 4 & $60,344,071$ & 71,206 & 71,205 & $\mathrm{t} / \mathrm{o}$ & $(86.66+0.39) 87.05$ & $(92.50+0.39)$ & 92.89 & $\mathrm{t} / \mathrm{o}$ & $(86.66+3.79)$ & 90.45 & $(92.50+3.79)$ & 96.25 \\
\hline
\end{tabular}


Table 7. $\mathrm{SWP}_{1}$

\begin{tabular}{|c|c|c|c|c|c|c|c|c|c|c|c|c|}
\hline \multirow[b]{2}{*}{$|M|$} & \multicolumn{3}{|c|}{ BES size } & \multicolumn{5}{|c|}{ Solving times (bigstep, default) } & \multicolumn{4}{|c|}{ Solving times (bigstep, no optimizations) } \\
\hline & original & $\sim$ & $\sim_{i i}$ & original & & $\sim$ & & $\sim_{i i}$ & original & $\sim$ & & $\sim_{i i}$ \\
\hline \multicolumn{13}{|c|}{ Property I } \\
\hline 1 & 684 & 5 & 2 & 0.00 & $(0.00+0.00)$ & 0.00 & $(0.00+0.00)$ & 0.00 & 0.00 & $(0.00+0.00) 0.00$ & $(0.00+0.00)$ & 0.00 \\
\hline 2 & 1,946 & 5 & 2 & 0.00 & $(0.00+0.00)$ & 0.00 & $(0.00+0.00)$ & 0.00 & 0.00 & $(0.00+0.00) 0.00$ & $(0.00+0.00)$ & 0.00 \\
\hline 3 & 3,788 & 5 & 2 & 0.01 & $(0.00+0.00)$ & 0.00 & $(0.00+0.00)$ & 0.00 & 0.01 & $(0.00+0.00) 0.00$ & $(0.00+0.00)$ & 0.00 \\
\hline 4 & 6,210 & 5 & 2 & 0.01 & $(0.00+0.00)$ & 0.00 & $(0.00+0.00)$ & 0.00 & 0.02 & $(0.00+0.00) 0.00$ & $(0.00+0.00)$ & 0.00 \\
\hline 5 & 9,212 & 5 & 2 & 0.02 & $(0.00+0.00)$ & 0.00 & $(0.00+0.00)$ & 0.00 & 0.04 & $(0.00+0.00) 0.00$ & $(0.00+0.00)$ & 0.00 \\
\hline 6 & 12,794 & 5 & 2 & 0.04 & $(0.00+0.00)$ & 0.00 & $(0.00+0.00)$ & 0.00 & 0.06 & $(0.00+0.00) 0.00$ & $(0.00+0.00)$ & 0.00 \\
\hline 7 & 16,956 & 5 & 2 & 0.05 & $(0.00+0.00)$ & 0.00 & $(0.01+0.00)$ & 0.01 & 0.08 & $(0.00+0.00) 0.00$ & $(0.01+0.00)$ & 0.01 \\
\hline 8 & 21,698 & 5 & 2 & 0.06 & $(0.00+0.00)$ & 0.00 & $(0.01+0.00)$ & 0.01 & 0.12 & $(0.00+0.00) 0.00$ & $(0.01+0.00)$ & 0.01 \\
\hline
\end{tabular}

Property II

\begin{tabular}{rrrllllllllllll}
\hline 1 & 1,408 & 566 & 7 & 0.00 & $(0.00+0.00)$ & 0.00 & $(0.00+0.00)$ & 0.00 & 0.00 & $(0.00+0.00)$ & 0.00 & $(0.00+0.00)$ & 0.00 \\
2 & 3,962 & 566 & 7 & 0.00 & $(0.00+0.00)$ & 0.00 & $(0.00+0.00)$ & 0.00 & 0.00 & $(0.00+0.00)$ & 0.00 & $(0.00+0.00)$ & 0.00 \\
3 & 7,664 & 566 & 7 & 0.01 & $(0.00+0.00)$ & 0.00 & $(0.00+0.00)$ & 0.00 & 0.02 & $(0.00+0.00)$ & 0.00 & $(0.00+0.00)$ & 0.00 \\
4 & 12,514 & 566 & 7 & 0.02 & $(0.02+0.00)$ & 0.02 & $(0.01+0.00)$ & 0.01 & 0.03 & $(0.02+0.00)$ & 0.02 & $(0.01+0.00)$ & 0.01 \\
5 & 18,512 & 566 & 7 & 0.05 & $(0.02+0.00)$ & 0.02 & $(0.01+0.00)$ & 0.01 & 0.05 & $(0.02+0.00)$ & 0.02 & $(0.01+0.00)$ & 0.01 \\
6 & 25,658 & 566 & 7 & 0.08 & $(0.03+0.00)$ & 0.03 & $(0.01+0.00)$ & 0.01 & 0.08 & $(0.03+0.00)$ & 0.03 & $(0.01+0.00)$ & 0.01 \\
7 & 33,952 & 566 & 7 & 0.11 & $(0.03+0.00)$ & 0.03 & $(0.02+0.00)$ & 0.02 & 0.12 & $(0.03+0.00)$ & 0.03 & $(0.02+0.00)$ & 0.02 \\
8 & 43,394 & 566 & 7 & 0.14 & $(0.02+0.00)$ & 0.02 & $(0.01+0.00)$ & 0.01 & 0.16 & $(0.02+0.00)$ & 0.02 & $(0.01+0.00)$ & 0.01 \\
\hline
\end{tabular}

Property III

\begin{tabular}{|c|c|c|c|c|c|c|c|c|c|c|c|}
\hline 1 & 840 & 386 & 386 & 0.00 & $(0.01+0.00)$ & 0.01 & $(0.01+0.00) 0.01$ & 0.00 & $(0.01+0.00)$ & 0.01 & $(0.01+0.00) 0.01$ \\
\hline 2 & 2,162 & 866 & 866 & 0.00 & $(0.01+0.00)$ & 0.01 & $(0.01+0.00) 0.01$ & 0.02 & $(0.01+0.00)$ & 0.01 & $(0.01+0.00) 0.01$ \\
\hline 3 & 4,064 & 866 & 866 & 0.01 & $(0.02+0.00)$ & 0.02 & $(0.02+0.00) 0.02$ & 0.04 & $(0.02+0.00)$ & 0.02 & $(0.02+0.00) 0.02$ \\
\hline 4 & 6,546 & 866 & 866 & 0.01 & $(0.01+0.00)$ & 0.01 & $(0.01+0.00) 0.01$ & 0.09 & $(0.01+0.00)$ & 0.01 & $(0.01+0.01) 0.02$ \\
\hline 5 & 9,608 & 866 & 866 & 0.03 & $(0.01+0.00)$ & 0.01 & $(0.02+0.00) 0.02$ & 0.15 & $(0.01+0.00)$ & 0.01 & $(0.02+0.00) 0.02$ \\
\hline 6 & 13,250 & 866 & 866 & 0.04 & $(0.02+0.00)$ & 0.02 & $(0.02+0.00) 0.02$ & 0.22 & $(0.02+0.01)$ & 0.03 & $(0.02+0.00) 0.02$ \\
\hline . & 17,472 & 866 & 866 & 0.07 & $(0.03+0.00)$ & 0.03 & $(0.02+0.00) 0.02$ & 0.32 & $(0.03+0.00)$ & 0.03 & $(0.02+0.00) 0.02$ \\
\hline 8 & 22,274 & 866 & 866 & 0.09 & $(0.04+0.00)$ & 0.04 & $(0.04+0.00) 0.04$ & 0.41 & $(0.04+0.00)$ & 0.04 & $(0.04+0.00) 0.04$ \\
\hline
\end{tabular}

Property IV

\begin{tabular}{|c|c|c|c|c|c|c|c|c|c|c|}
\hline 1 & 842 & 388 & 388 & 0.00 & $(0.01+0.00)$ & 0.01 & $(0.02+0.00) 0.02$ & 0.00 & $(0.01+0.00) 0.01$ & $(0.02+0.00) 0.02$ \\
\hline 2 & 4,327 & 869 & 868 & 0.01 & $(0.01+0.00)$ & 0.01 & $(0.02+0.00) 0.02$ & 0.04 & $(0.01+0.00) 0.01$ & $(0.02+0.00) 0.02$ \\
\hline 3 & 12,196 & 869 & 868 & 0.04 & $(0.02+0.00)$ & 0.02 & $(0.03+0.00) 0.03$ & 0.16 & $(0.02+0.01) 0.03$ & $(0.03+0.00) 0.03$ \\
\hline 4 & 26,189 & 869 & 868 & 0.10 & $(0.03+0.00)$ & 0.03 & $(0.04+0.00) 0.04$ & 0.42 & $(0.03+0.00) 0.03$ & $(0.04+0.00) 0.04$ \\
\hline & 48,046 & 869 & 868 & 0.22 & $(0.05+0.00)$ & 0.05 & $(0.03+0.00) 0.03$ & 0.83 & $(0.05+0.00) 0.05$ & $(0.03+0.00) 0.03$ \\
\hline 5 & 79,507 & 869 & 868 & 0.38 & $(0.07+0.00)$ & 0.07 & $(0.05+0.00) 0.05$ & 1.48 & $(0.07+0.00) 0.07$ & $(0.05+0.00) 0.05$ \\
\hline 7 & 122,312 & 869 & 868 & 0.70 & $(0.11+0.00)$ & 0.11 & $(0.08+0.00) 0.08$ & 2.42 & $(0.11+0.00) 0.11$ & $(0.08+0.00) 0.08$ \\
\hline 8 & 178,201 & 869 & 868 & 0.93 & $(0.17+0.00)$ & 0.17 & $(0.12+0.00) 0.12$ & 3.68 & $(0.17+0.00) 0.17$ & $(0.12+0.00) 0.12$ \\
\hline
\end{tabular}

\begin{tabular}{rrrllllllllllll}
\hline \multicolumn{10}{c}{ Property V } \\
\hline 1 & 2,372 & 1,090 & 1,090 & 0.01 & $(0.02+0.00)$ & 0.02 & $(0.02+0.00)$ & 0.02 & 0.03 & $(0.02+0.01)$ & 0.03 & $(0.02+0.01)$ & 0.03 \\
2 & 12,749 & 2,489 & 2,488 & 0.06 & $(0.02+0.00)$ & 0.02 & $(0.02+0.01)$ & 0.03 & 0.17 & $(0.02+0.03)$ & 0.05 & $(0.02+0.02)$ & 0.04 \\
3 & 36,414 & 2,489 & 2,488 & 0.20 & $(0.04+0.01)$ & 0.05 & $(0.03+0.00)$ & 0.03 & 0.57 & $(0.04+0.03)$ & 0.07 & $(0.03+0.03)$ & 0.06 \\
4 & 78,647 & 2,489 & 2,488 & 0.48 & $(0.08+0.01)$ & 0.09 & $(0.09+0.01)$ & 0.10 & 1.32 & $(0.08+0.02)$ & 0.10 & $(0.09+0.03)$ & 0.12 \\
5 & 144,728 & 2,489 & 2,488 & 0.90 & $(0.14+0.00)$ & 0.14 & $(0.16+0.00)$ & 0.16 & 2.94 & $(0.14+0.02)$ & 0.16 & $(0.16+0.02)$ & 0.18 \\
6 & 239,937 & 2,489 & 2,488 & 1.56 & $(0.25+0.01)$ & 0.26 & $(0.27+0.00)$ & 0.27 & 4.42 & $(0.25+0.02)$ & 0.27 & $(0.27+0.02)$ & 0.29 \\
7 & 369,554 & 2,489 & 2,488 & 2.39 & $(0.28+0.01)$ & 0.29 & $(0.31+0.01)$ & 0.32 & 6.94 & $(0.28+0.03)$ & 0.31 & $(0.31+0.03)$ & 0.34 \\
8 & 538,859 & 2,489 & 2,488 & 3.67 & $(0.56+0.01)$ & 0.57 & $(0.61+0.01)$ & 0.62 & 10.45 & $(0.56+0.02)$ & 0.58 & $(0.61+0.02)$ & 0.63 \\
\hline
\end{tabular}


Table 8. $\mathrm{SWP}_{2}$

\begin{tabular}{|c|c|c|c|c|c|c|c|c|c|c|c|c|}
\hline \multirow[b]{2}{*}{$|M|$} & \multicolumn{3}{|c|}{ BES size } & \multicolumn{4}{|c|}{ Solving times (bigstep, default) } & \multicolumn{5}{|c|}{ Solving times (bigstep, no optimizations) } \\
\hline & original & $\sim$ & $\sim_{i i}$ & original & $\sim$ & & $\sim_{i i}$ & original & & $\sim$ & & $\sim_{i i}$ \\
\hline \multicolumn{13}{|c|}{ Property I } \\
\hline 1 & 9,082 & 5 & 2 & 0.02 & $(0.00+0.00) 0.00$ & $(0.00+0.00)$ & 0.00 & 0.04 & $(0.00+0.00)$ & 0.00 & $(0.00+0.00)$ & 0.00 \\
\hline 2 & 71,090 & 5 & 2 & 0.29 & $(0.00+0.00) 0.00$ & $(0.02+0.00)$ & 0.02 & 0.48 & $(0.00+0.00)$ & 0.00 & $(0.02+0.00)$ & 0.02 \\
\hline 3 & 269,282 & 5 & 2 & 1.34 & $(0.05+0.00) 0.05$ & $(0.11+0.00)$ & 0.11 & 2.10 & $(0.05+0.00)$ & 0.05 & $(0.11+0.00)$ & 0.11 \\
\hline 4 & 728,386 & 5 & 2 & 3.92 & $(0.09+0.00) 0.09$ & $(0.25+0.00)$ & 0.25 & 6.62 & $(0.09+0.00)$ & 0.09 & $(0.25+0.00)$ & 0.25 \\
\hline 5 & $1,614,602$ & 5 & 2 & 9.17 & $(0.17+0.00) 0.17$ & $(0.62+0.00)$ & 0.62 & 13.99 & $(0.17+0.00)$ & 0.17 & $(0.62+0.00)$ & ) 0.62 \\
\hline \multicolumn{13}{|c|}{ Property II } \\
\hline 1 & 18,138 & 2,268 & 7 & 0.05 & $(0.03+0.00) 0.03$ & $(0.01+0.00)$ & 0.01 & 0.04 & $(0.03+0.00)$ & 0.03 & $(0.01+0.00)$ & 0.01 \\
\hline 2 & 139,698 & 2,268 & 7 & 0.54 & $(0.13+0.00) 0.13$ & $(0.07+0.00)$ & 0.07 & 0.55 & $(0.13+0.00)$ & 0.13 & $(0.07+0.00)$ & 0.07 \\
\hline 3 & 525,026 & 2,268 & 7 & 2.27 & $(0.35+0.00) 0.35$ & $(0.42+0.00)$ & 0.42 & 2.32 & $(0.35+0.00)$ & 0.35 & $(0.42+0.00)$ & 0.42 \\
\hline 4 & $1,413,954$ & 2,268 & 7 & 6.66 & $(0.93+0.00) 0.93$ & $(0.69+0.00)$ & 0.69 & 6.79 & $(0.93+0.00)$ & 0.93 & $(0.69+0.00)$ & 0.69 \\
\hline 5 & $3,125,802$ & 2,268 & 7 & 16.01 & $(2.05+0.00) 2.05$ & $(1.59+0.00)$ & 1.59 & 16.39 & $(2.05+0.00)$ & 2.05 & $(1.59+0.00)$ & ) 1.59 \\
\hline \multicolumn{13}{|c|}{ Property III } \\
\hline 1 & 11,482 & 2,013 & 2,013 & 0.04 & $(0.03+0.00) 0.03$ & $(0.03+0.00)$ & 0.03 & 0.10 & $(0.03+0.00)$ & 0.03 & $(0.03+0.01)$ & 0.04 \\
\hline 2 & 80,146 & 9,097 & 9,097 & 0.44 & $(0.07+0.02) 0.09$ & $(0.08+0.03)$ & 0.11 & 1.22 & $(0.07+0.09)$ & 0.16 & $(0.08+0.08)$ & 0.16 \\
\hline 3 & 291,842 & 9,097 & 9,097 & 1.85 & $(0.27+0.02) 0.29$ & $(0.28+0.03)$ & 0.31 & 6.12 & $(0.27+0.09)$ & 0.36 & $(0.28+0.09)$ & 0.37 \\
\hline 4 & 773,890 & 9,097 & 9,097 & 5.21 & $(0.65+0.03) 0.68$ & $(0.71+0.03)$ & 0.74 & 17.83 & $(0.65+0.09)$ & 0.74 & $(0.71+0.09)$ & 0.80 \\
\hline 5 & $1,695,082$ & 9,097 & 9,097 & 11.94 & $(1.47+0.03) 1.50$ & $(1.59+0.03)$ & 1.62 & 46.84 & $(1.47+0.09)$ & 1.56 & $(1.59+0.09)$ & ) 1.68 \\
\hline \multicolumn{13}{|c|}{ Property IV } \\
\hline 1 & 11,484 & 2,015 & 2,015 & 0.04 & $(0.03+0.00) 0.03$ & $(0.03+0.00)$ & 0.03 & 0.10 & $(0.03+0.01)$ & 0.04 & $(0.03+0.01)$ & 0.04 \\
\hline 2 & 160,295 & 9,100 & 9,099 & 0.95 & $(0.23+0.02) 0.25$ & $(0.22+0.03)$ & 0.25 & 2.52 & $(0.23+0.09)$ & 0.32 & $(0.22+0.08)$ & 0.30 \\
\hline 3 & 875,530 & 9,100 & 9,099 & 5.72 & $(0.77+0.02) 0.79$ & $(0.82+0.02)$ & 0.84 & 17.27 & $(0.77+0.09)$ & 0.86 & $(0.82+0.09)$ & 0.91 \\
\hline 4 & $3,095,565$ & 9,100 & 9,099 & 22.17 & $(2.65+0.02) 2.67$ & $(3.22+0.03)$ & 3.25 & 72.31 & $(2.65+0.09)$ & 2.74 & $(3.22+0.08)$ & 3.30 \\
\hline 5 & $8,475,416$ & 9,100 & 9,099 & 69.42 & $(7.49+0.03) 7.52$ & $(8.06+0.03)$ & 8.09 & 218.20 & $(7.49+0.09)$ & 7.58 & $(8.06+0.09)$ & 8.15 \\
\hline \multicolumn{13}{|c|}{ Property V } \\
\hline 1 & 31,360 & 5,500 & 5,500 & 0.49 & $(0.07+0.06) 0.13$ & $(0.07+0.07)$ & 0.14 & 0.85 & $(0.07+0.11)$ & 0.18 & $(0.07+0.11)$ & 0.18 \\
\hline 2 & 456,037 & 25,354 & 25,353 & 9.40 & $(0.58+0.32) 0.90$ & $(0.90+0.33)$ & 1.23 & 30.67 & $(0.58+0.57)$ & 1.15 & $(0.90+0.56)$ & ) 1.46 \\
\hline 3 & $2,520,258$ & 25,354 & 25,353 & 67.10 & $(3.69+0.34) 4.03$ & $(2.87+0.34)$ & 3.21 & 167.57 & $(3.69+0.58)$ & 4.27 & $(2.87+0.57)$ & 3.44 \\
\hline 4 & $8,957,959$ & 25,354 & 25,353 & 208.51 & $(8.82+0.31) 9.13$ & $(9.84+0.32) 1$ & 10.16 & $\mathrm{t} / \mathrm{o}$ & $(8.82+0.58)$ & 9.40 & $(9.84+0.58)$ & 10.42 \\
\hline 5 & $24,599,908$ & 25,354 & 25,353 & $\mathrm{t} / \mathrm{o}$ & $(27.44+0.33) 27.77$ & $(44.52+0.33) 4$ & 44.85 & $\mathrm{t} / \mathrm{o}$ & $(27.44+0.57) 2$ & 28.01 & $(44.52+0.57)$ & 45.09 \\
\hline
\end{tabular}

Table 9. $\mathrm{SWP}_{3}$

\begin{tabular}{|c|c|c|c|c|c|c|c|c|c|c|c|}
\hline \multirow[b]{2}{*}{$|M|$} & \multicolumn{3}{|c|}{ BES size } & \multicolumn{4}{|c|}{ Solving times (bigstep, default) } & \multicolumn{4}{|c|}{ Solving times (bigstep, no optimizations) } \\
\hline & original & $\sim$ & $\sim_{i i}$ & original & $\sim$ & & $\sim_{i i}$ & original & $\sim$ & & $\sim_{i i}$ \\
\hline \multicolumn{12}{|c|}{ Property I } \\
\hline 1 & 54,464 & 5 & 2 & 0.20 & $(0.01+0.00) 0.01$ & $(0.02+0.00)$ & 0.02 & 0.35 & $(0.01+0.00) 0.01$ & $(0.02+0.00)$ & 0.02 \\
\hline 2 & $1,187,906$ & 5 & 2 & 6.66 & $(0.20+0.00) 0.20$ & $(0.44+0.00)$ & 0.44 & 10.17 & $(0.20+0.00) 0.20$ & $(0.44+0.00)$ & 0.44 \\
\hline \multicolumn{12}{|c|}{ Property II } \\
\hline 1 & 107,300 & 7,018 & 7 & 0.39 & $(0.10+0.01) 0.11$ & $(0.04+0.00)$ & 0.04 & 0.39 & $(0.10+0.01) 0.11$ & $(0.04+0.00)$ & 0.04 \\
\hline 2 & $2,299,394$ & 7,018 & 7 & 11.33 & $(2.62+0.01) 2.63$ & $(1.57+0.00)$ & 1.57 & 11.39 & $(2.62+0.01) 2.63$ & $(1.57+0.00)$ & 1.57 \\
\hline \multicolumn{12}{|c|}{ Property III } \\
\hline 1 & 69,500 & 6,790 & 6,790 & 0.41 & $(0.09+0.02) 0.11$ & $(0.11+0.02)$ & 0.13 & 0.78 & $(0.09+0.05) 0.14$ & $(0.11+0.04)$ & 0.15 \\
\hline 2 & $1,345,634$ & 69,915 & 69,915 & 10.14 & $(2.43+0.39) 2.82$ & $(2.58+0.38)$ & 2.96 & 21.91 & $(2.43+0.85) 3.28$ & $(2.58+0.85)$ & 3.43 \\
\hline \multicolumn{12}{|c|}{ Property IV } \\
\hline 1 & 69,502 & 6,792 & 6,792 & 0.40 & $(0.09+0.02) 0.11$ & $(0.07+0.02)$ & 0.09 & 0.78 & $(0.09+0.04) 0.13$ & $(0.07+0.05)$ & 0.12 \\
\hline 2 & $2,691,271$ & 69,918 & 69,917 & 20.72 & $(4.76+0.39) 5.15$ & $(5.79+0.39)$ & 6.18 & 41.42 & $(4.76+0.90) 5.66$ & $(5.79+0.89)$ & 6.68 \\
\hline \multicolumn{12}{|c|}{ Property V } \\
\hline$\overline{1}$ & 187,828 & 18,346 & 18,346 & 9.63 & $(0.30+0.46) 0.76$ & $(0.33+0.46)$ & 0.79 & 7.95 & $(0.30+0.59) 0.89$ & $(0.33+0.59)$ & $\overline{0.92}$ \\
\hline 2 & $7,558,853$ & 192,664 & 192,663 & $\mathrm{t} / \mathrm{o}$ & $(19.70+7.58) 27.28$ & $(20.03+7.58) 2$ & 27.61 & 289.26 & $(19.70+11.29) 30.99$ & $(20.03+11.31)$ & 31.34 \\
\hline
\end{tabular}

